\title{
Index to scientific names and nomenclatural checklist of Trypetheliaceae
}

Accepted names are in bold face, synonyms in italics (with accepted names indicated in parentheses), and other names not belonging in Trypetheliaceae in normal font. Main page entries for accepted taxa are in bold face; entries referring to figure plates (f), key entries (k), phylograms (p), and tables ( $\mathrm{t}$ ) are in italics; other text and synonymy entries are in normal font. SA, SB, SC, and SX refer to Supplemental files references in Lücking et al., pp. 739-762 (SA-SC) and Aptroot et al., pp. 983994 (SX).

acroleucum, Astrothelium (=Astrothelium eustomum): 859. acrophaeum, Astrothelium: 783(t), 808(k), 821, 838(f), $\mathrm{SX}(\mathrm{t})$.

acrophaeum, Pleurotrema (=Astrothelium acrophaeum): 821 . acrophaeum, Trypethelium (=Astrothelium acrophaeum): 821 . adacta, Pyrenula: 976.

aenascens, Astrothelium: 611, 612(f), 624, 714, 729(t), 730(p), 731, 732, 736, 798(k), 824(f), 828(f), $\mathrm{SX}(\mathrm{t})$.

aenea, Pseudopyrenula (=Astrothelium aeneum): 821.

aenea, Verrucaria (=Astrothelium aeneum): 821 .

aeneoides, Astrothelium: 701, 714, 715(f), 799(k), 826(f), SX(t).

aeneum, Astrothelium: 611, 641, 671, 687(t), 701, 702, 714, 724, 725, 743(t), 752(p), 755, 766(f), 781, 784, 785, 798(k), 821，825(f)，866，SA(a)，SC(p), $\mathrm{SX}(\mathrm{t})$.

aeneum, Trypethelium (=Astrothelium aeneum): 821, 860 . aenoides, Astrothelium: 611.

africanum, Astrothelium (= Lithothelium obtectum): 977. alata, Bogoriella: 688(t), 904(k), 905(k), 905, SX(t). alata, Mycomicrothelia (=Bogoriella alata): 905.

alba, Microthelia (=Bogoriella obovata): 913.

albida, Arthopyrenia (= Polymeridium albidum): 941.

albidella, Microthelia (=Bogoriella thelena): 916 .

albidoporum, Bathelium: $800(\mathrm{k}), 896(\mathrm{k}), 897(\mathrm{f}), \mathbf{8 9 7}$, $\mathrm{SX}(\mathrm{t})$.

albidoporum, Trypethelium (= Bathelium albidoporum): 897 .

albidoreagens, Polymeridium: 929(f), 935(k), 938, $\mathrm{SX}(\mathrm{t})$.

albidovarians, Polymeridium: 932(f), 934(k), 940, $\mathrm{SX}(\mathrm{t})$.

albidum, Polymeridium: 688(t), 936(k), 939(f), 941, $\mathrm{SX}(\mathrm{t})$.

alboatra var. detergens, Verrucaria (=Constrictolumina cinchonae): 919.

albocinerea, Arthopyrenia (= Polymeridium albocinereum): 942.

albocinerea, Verrucaria (= Polymeridium albocinereum): 942.

\footnotetext{
${ }^{*}$ The original version of this index was published with the incorrect running head. A notice detailing this has been published and the error rectified in the online PDF and HTML copies.
}

albocinereum, Polymeridium: $747(\mathrm{t}), 750(\mathrm{p}), 785$, 937(k), 941(f), 942, SA(a), SX(t).

alboflavescens, Polymeridium: 683, 685, 936(k), $940(\mathrm{f})$, 942, SX (t).

albonitens, Pseudopyrenula (= Pseudopyrenula diluta): 957. albopruinosum, Polymeridium: 688(t), 929(f), 934(k), 942, SX $(\mathrm{t})$.

albopruinosum, Trypethelium (= Polymeridium albopruinosum): 942.

alboverruca, Laurera (=Astrothelium alboverrucum): 823.

alboverrucoides, Astrothelium: 611, 612(f), 814(k), 844(f), SX(t).

alboverrucum, Astrothelium: $815(\mathrm{k}), \mathbf{8 2 3}, 845(\mathrm{f}), \mathrm{SX}(\mathrm{t})$ album var. verrucarioides, Pyrenastrum (= Polymeridium catapastum): 944.

album, Campylothelium (=Dictyomeridium proponens): 926. alpina, Heufleria (= non-lichenized fungus): 977.

amazonum, Astrothelium: 644, 687(t), 818(k), 825, 847(f), SX(t).

amazonum, Cryptothelium (=Astrothelium amazonum): 826 . ambigua, Laurera (=Astrothelium ambiguum): 828 .

ambiguum, Astrothelium: 813(k), 828, 843(f), SX(t). ambiguum, Meristosporum (=Astrothelium ambiguum): 828 . americana, Pseudopyrenula: 633, 955(k), SX(t). amyloideum, Polymeridium: 774(f), 932(f), 934(k), 943, SX (t).

amylospora, Polyblastiopsis (= Dictyomeridium amylosporum): 923.

amylospora, Thelenella (=Dictyomeridium amylosporum): 923. amylosporum, Astrothelium: 663(f), 664(f), 665, 687(t), 815(k), 845(f), SX(t).

amylosporum, Campylothelium (=Dictyomeridium amylosporum): 923.

amylosporum, Dictyomeridium: 923(k), 923, 924(f), $\mathrm{SX}(\mathrm{t})$.

amylosporum, Polymeridium (=Dictyomeridium amylosporum): 923.

anacardii, Trypethelium (= Trypethelium eluteriae): 964 .

analepta, Arthopyrenia (=Arthopyrenia analepta): 917.

andamanica, Laurera (=Astrothelium meristosporum): 831, 871.

andamanicum, Astrothelium: 658, 819(k), 830, 848(f), $\mathrm{SX}(\mathrm{t})$.

andamanicum, Cryptothelium (=Astrothelium andamanicum): 831.

andamanicum, Trypethelium (= Trypethelium epileucodes): 967(f), 969.

angulata, Distothelia: 779(f), 903(k), 927(k), 927, SX(t). annonacea, Bogoriella: 905(k), 907, SX(t).

annonacea, Microthelia (=Microthelia annonacea): 908.

annonacea, Mycomicrothelia (=Mycomicrothelia annonacea): 908.

annulare var. detrusum, Trypethelium (=Astrothelium annulare): 834, 836.

annulare var. meiophorum, Trypethelium (=Astrothelium meiophorum): 870, 871 . 
annulare var. subdepressum, Trypethelium (= Pyrenula oleagina): 978.

annulare, Astrothelium: 627, 687(t), 719, 720, 802(k), 831(f), 833, SX(t).

annulare, Trypethelium (=Astrothelium annulare): 833 .

annularis var. tuberculosa, Pseudopyrenula (=Astrothelium tuberculosum): 893.

annularis, Pseudopyrenula (=Astrothelium annulare): 833. annularis, Pyrenula (=Astrothelium annulare): 833.

annularis, Verrucaria (=Astrothelium annulare): 833.

anoista, Verrucaria (= Dictyomeridium proponens): 926.

anoistum, Anthracothecium (= Dictyomeridium proponens): 926. anoistum, Campylothelium (=Dictyomeridium proponens): 926. anomala, Pyrenula: 976, 977, 978.

anomalum $\mathrm{f}$. leucostomum, Trypethelium (= Trypethelium sphaerocephalum): 971.

anomalum var. obscurescens, Trypethelium (=Pyrenula anomala): 978.

anomalum, Trypethelium (=Pyrenula anomala): 978.

anoptella, Clathroporina (=Astrothelium meristosporum): 871 . anoptella, Polyblastiopsis (=Astrothelium meristosporum): 871. anoptella, Verrucaria (=Astrothelium meristosporum): 841(f), 871.

Anthracothecium: 977.

antoniae, Pseudopyrenula (=Astrothelium cinereorosellum): 853. antoniae, Pyrenula (=Astrothelium cinereorosellum): 853.

antoniae, Verrucaria (=Astrothelium cinereorosellum): 822(f), 853.

apposita, Arthopyrenia (=Bogoriella apposita): 908.

apposita, Bogoriella: 688(t), 905(k), 907(f), 908, 908, $\mathrm{SX}(\mathrm{t})$.

apposita, Microthelia (=Bogoriella apposita): 908.

apposita, Mycomicrothelia (=Bogoriella apposita): 908.

apposita, Verrucaria (=Bogoriella apposita): 908.

Aptrootia: 649, 741, 749(p), 749, 751, 755, 778, 788(k), 789, 789.

araucariae, Pseudopyrenula (= Pseudopyrenula subnudata): 957(f), 960.

arborea, Ochrolechia: 662 .

Architrypethelium: $641,683,741,749$ (p), 751, 755, 767, 778, 788(k), 792, 792.

arctecincta, Pyrenula: 978.

areolatum, Trypethelium (= Trypethelium eluteriae): 964 .

Arthopyrenia: 741, 748, 751, 753, 756, 767, 769, 778, $786,917$.

assimile, Trypethelium (= Trypethelium eluteriae): 964 .

astroidella, Laurera (= non-lichenized fungus): 977.

astroideum, Trypethelium: 682(f), 684(f), 685, 686, 689(t), 773(f), 780(f), 963(k), 965(f), SX(t).

Astrothelium: 640, 658, 690, 710, 711, 719, 723, 727, $728,730(\mathrm{p}), 741,749(\mathrm{p}), 749,751,752(\mathrm{p}), 753$, 754(p), 755, 757, 758, 766(f), 768, 772, 775, 781, 782, 786, 787, 788(k), 789(k), 792, 795, 796(k), 895, 961, 962, 972.

atroalba, Arthopyrenia (=Polymeridium contendens): 945.

atroalba, Pseudopyrenula (= Pseudopyrenula diluta): 957(f), 957.

aurantia, Verrucaria (=Astrothelium amazonum): 826.

aurantiaca, Laurera (=Astrothelium aurantiacum): 837.

aurantiacocinereum, Astrothelium: 641, 642(f), 780(f), 798(k), 824(f), SX(t).

aurantiacum, Astrothelium: 814(k), 836, 843(f), SX(t). aurata, Laurera (=Astrothelium auratum): 839.

auratum, Astrothelium: 812(k), 839, 842(f), SX(t).

aureomaculata, Pseudopyrenula (=Astrothelium aureomaculatum): 842.

aureomaculatum, Astrothelium: 804(k), 833(f), 841, $\mathrm{SX}(\mathrm{t})$.

aureomaculatum, Trypethelium (=Astrothelium aureomaculatum): 842 .

australiense, Anthracothecium: 977.

austroafricanum, Bathelium: 799(k), 896(k), 899, SX(t). austroafricanum, Trypethelium (=Bathelium austroafricanum): 899.

awajiensis, Pseudopyrenula (=Pyrenula awajiensis): 977.

awajiensis, Pyrenula: 977.

baccharidis, Didymosphaeria (=Bogoriella subfallens): 915.

balia, Pseudopyrenula (= Pyrenula balia): 977.

balia, Pyrenula: 977.

bambusicola, Polymeridium: 937(k), 941(f), 943, SX(t).

basilica, Porina (=Astrothelium basilicum): 845 .

basilica, Pseudopyrenula (=Astrothelium basilicum): 844 .

basilica, Verrucaria (=Astrothelium basilicum): 844.

basilicum, Astrothelium: 803(k), 831(f), 844, SX(t).

basilicum, Trypethelium (=Astrothelium basilicum): 845 .

Bathelium: 654, 690, 741, 749(p), 749, 752(p), 753, $755,757,775,777,778,782,786,787,788(\mathrm{k}), 789(\mathrm{k})$, 852, 877, 884, 895, 931, 962.

bengoana, Pseudopyrenula (=Polymeridium bengoanum): 943.

bengoanum, Polymeridium: 929(f), 934(k), 943, SX(t). benguelense, Bathelium (=Marcelaria benguelensis): 928.

benguelensis, Laurera (= Marcelaria benguelensis): 928.

benguelensis, Marcelaria: 781, 785, 928(k), 928, 929(f), $\mathrm{SX}(\mathrm{t})$.

bicincta, Pseudopyrenula (= Nigrovothelium tropicum): 931.

bicolor var. pyrenuloides, Trypethelium (=Astrothelium phlyctaena): 878.

bicolor, Astrothelium: 674, 687(t), 697, 743(t), 752(p), 783(t), 784, 785, 801(k), 828(f), 847, 989, SA(a), SX(t).

bicolor, Bathelium (=Astrothelium bicolor): 847.

bicolor, Trypethelium (=Astrothelium bicolor): 847 .

bifera, Arthopyrenia (=Constrictolumina malaccitula): 921.

biloculare, Polymeridium: 935(k), 939(f), 943, SX(t). bispora, Laurera (=Astrothelium andamanicum): 831 .

bivelum, Astrothelium: 694, 695(f), 708(f), 807(k), 836(f), SX(t).

Bogoriella: $748,749(\mathrm{p}), 750(\mathrm{p}), 751,757,758,767$, 769, 778, 782, 786, 787(k), 902, 953, 987.

boliviense, Bathelium: 675(f), 676(f), 677, 678, 688(t), 896(k), 897(f), SX(t).

boninense, Trypethelium (=Astrothelium bicolor): 848 .

Botryosphaeria: 976.

brachysporum, Polymeridium (=Astrothelium porosum): 879.

brachysporum, Trypethelium (= Astrothelium porosum): 822(f), 879.

brisbanensis, Microthelia (=Bogoriella obovata): 913.

buckii, Astrothelium: 720, 798(k), 824(f), 850, SX(t).

buckii, Trypethelium (=Astrothelium buckii): 850 .

bullatum, Astrothelium: 663(f), 664(f), 665, 666, 687(t), $820(\mathrm{k}), 849(\mathrm{f}), \mathrm{SX}(\mathrm{t})$.

bullatum, Nigrovothelium: 655, 656(f), 746(t), 747(t), $750(\mathrm{p}), 756,801(\mathrm{k}), 931(\mathrm{k}), 932(\mathrm{f}), \mathrm{SA}(\mathrm{a}), S B(\mathrm{p})$, $\mathrm{SC}(\mathrm{p}), \mathrm{SX}(\mathrm{t})$.

buxi, Julella: 751 . 
calospora var. rhodocheila, Pseudopyrenula (=Astrothelium calosporum): 851.

calospora, Pseudopyrenula (=Astrothelium calosporum): 851 .

calosporum, Astrothelium: 774(f), 800(k), 827(f), 851, SX $(\mathrm{t})$.

calosporum, Trypethelium (=Astrothelium calosporum): 851 .

campylocartilagineum, Astrothelium: 658, $820(\mathrm{k})$, 849(f), 851, SX(t).

campylothelioides, Dictyomeridium: 923(k), 924(f), 924, SX(t).

campylothelioides, Polymeridium (= Polymeridium campylothelioides): 925.

Campylothelium (=Astrothelium ): 640, 707, 710, 741, 749, 753, 755, 757, 781, 782, 795, 796(k), 923.

captiosa, Bogoriella: 904(k), 907(f), 908, SX(t).

captiosa, Microthelia (=Bogoriella captiosa): 908.

captiosa, Mycomicrothelia (=Bogoriella captiosa): 908.

captiosa, Verrucaria (=Bogoriella captiosa): 908.

carassense, Astrothelium: 642(f), 643, 743(t), 752(p), 818(k), 847(f), SA(a), SX(t).

carolinianum, Bathelium: $896(\mathrm{k}), 897(\mathrm{f}), \mathbf{8 9 9}, \mathrm{SX}(\mathrm{t})$.

carolinianum, Trypethelium (=Bathelium carolinianum): 899 .

carrascoense, Astrothelium: 644, 663(f), 664(f), 666, 667, 687(t), 816(k), 846(f), SX(t).

cartilaginea, Pyrenula (=Astrothelium cartilagineum): 851 .

cartilagineum, Astrothelium: 687(t), 799(k), 850, 851, 851, SX(t).

cartilagineum, Campylothelium (=Astrothelium campylocartilagineum): 851.

cartilagineum, Trypethelium (=Astrothelium cartilagineum): 851.

cascarillae, Trypethelium (=Astrothelium phlyctaena): 877 . catapasta, Arthopyrenia (=Polymeridium catapastum): 944.

catapasta, Verrucaria (= Polymeridium catapastum): 944.

catapastoides, Polymeridium: 935(k), 939(f), 943, SX $(\mathrm{t})$.

catapastum, Polymeridium: $688(\mathrm{t}), 747(\mathrm{t}), 750(\mathrm{p})$, 769(f), 785, 929(f), 935(k), 944, SA(a), SX(t).

catervaria, Pseudopyrenula (=Astrothelium phlyctaena): 877.

catervaria, Pyrenula (= Astrothelium phlyctaena): 877.

catervaria, Verrucaria (=Astrothelium phlyctaena): 877.

catervarium var. rufescens, Trypethelium (=Astrothelium rufescens): 884.

catervarium, Spermatodium (=Astrothelium phlyctaena): 877.

catervarium, Trypethelium (=Astrothelium phlyctaena): 877 .

cecidiogenum, Astrothelium: 701, 743(t), 754(p), 766(f), 821(k), 849(f), 851, SA(a), SX(t).

cecidiogenum, Cryptothelium (=Astrothelium cecidiogenum): 852.

celatum, Trypethelium (=Astrothelium eustomum): 859 .

ceratina, Pseudopyrenula (=Astrothelium ceratinum): 852 .

ceratina, Pyrenula (=Astrothelium ceratinum): 852 .

ceratina, Verrucaria (=Astrothelium ceratinum): 852.

ceratinum, Astrothelium: 709, 771(f), 783(t), 801(k), 829(f), 852, SX(t).

ceratinum, Trypethelium (=Astrothelium ceratinum): 852 .

cerei, Pseudopyrenula: 954(k), 955, 956(f), SX(t).

ceylonicum, Trypethelium (= Trypethelium subeluteriae): 972 .

chapadense, Astrothelium: 816(k), 846(f), 852, SX(t).

chapadense, Meristosporum (=Astrothelium chapadense): 852 . chapadensis, Laurera (=Astrothelium chapadense): 852 . chiodectonoides, Trypethelium (=Pertusaria feeana): 978. chionea, Pseudopyrenula (=Polymeridium chioneum): 944. chionea, Pyrenula (= Polymeridium chioneum): 944.

chionea, Verrucaria (= Polymeridium chioneum): 944.

chioneum, Polymeridium: 936(k), 939(f), 944, SX(t).

chiquitana, Constrictolumina: 681, 682(f), 684(f), 688(t), 917(k), 918(f), SX(t).

chlorogastrica, Heufleria (= Phyllobathelium chlorogastricum): 851, 976.

chlorogastricum, Campylothelium (=Phyllobathelium chlorogastricum): 976.

chlorogastricum, Cryptothelium (= Phyllobathelium chlorogastricum): 976.

chlorogastricum, Phyllobathelium: 976.

chrysocarpa, Laurera (=Astrothelium meristosporum): 871 . chrysocarpum, Bathelium (=Astrothelium meristosporum): 871 . chrysoglypha, Laurera (=Astrothelium chrysoglyphum): 853. chrysoglypha, Thelenella (=Astrothelium chrysoglyphum): 853 . chrysoglyphum, Astrothelium: 813(k), 842(f), 853, $\mathrm{SX}(\mathrm{t})$.

chrysostomum, Astrothelium: $805(\mathrm{k}), 833(\mathrm{f}), 853$, $\mathrm{SX}(\mathrm{t})$.

chrysostomum, Trypethelium (=Astrothelium chrysostomum): 853.

cicatricosa, Glyphis: 976.

cinchonae, Arthopyrenia (=Constrictolumina cinchonae): 756, 919

cinchonae, Constrictolumina: $746(\mathrm{t}), 750(\mathrm{p}), \mathbf{7 5 6}$, 756, 784, 917, 918(k), 919(f), 919, SA(a), SX(t).

cinchonae, Verrucaria (=Constrictolumina cinchonae): 756, 919.

cinereoglaucescens, Pseudopyrenula (=Viridothelium cinereoglaucescens): 973

cinereoglaucescens, Trypethelium (=Viridothelium cinereoglaucescens): 973.

cinereoglaucescens, Viridothelium: $802(\mathrm{k}), 963(\mathrm{k})$, 973(k), 973, 974(f), SX(t).

cinereonigricans, Clathroporina (=Polymeridium cinereonigricans): 944.

cinereonigricans, Polymeridium: 937(k), 941(f), 944, SX(t).

cinereonigricans, Thelenella (=Polymeridium cinereonigricans): 944.

cinereorosellum, Astrothelium: 644, 646, 696, 743(t), 754(p), 755, 796(k), 822(f), 853, SA(a), SC(p), SX(t).

cinereorosellum, Trypethelium $(=$ Astrothelium cinereorosellum): 853 .

cinereum, Astrothelium: $820(\mathrm{k}), 849(\mathrm{f}), 854, \mathrm{SX}(\mathrm{t})$.

cinnabarinum, Trypethelium (= Pyrenula cruenta): 978 .

cinnamomeum, Astrothelium: 672, 673, 687(t), 727, 729(t), 730(p), 743(t), 752(p), 785, 795, 807(k), 836(f), 854, SA(a), SX(t).

cinnamomeum, Pyrenastrum (=Astrothelium cinnamomeum): 854.

cladosporioides, Cladosporium: 742, 743(t), SA.

clandestina, Polyblastia (=Astrothelium crassum): 855.

clandestinum, Astrothelium (=Astrothelium crassum): 855 .

clandestinum, Pyrenastrum (=Astrothelium crassum): 855 .

clandestinum, Trypethelium (=Astrothelium crassum): 855 .

clypeatum, Astrothelium: 612(f), 613, 802(k), 830(f), $\mathrm{SX}(\mathrm{t})$. 
coccifera var. cinereorubricosa, Didymosphaeria (=Bogoriella subfallens): 915.

coccifera, Didymosphaeria (=Bogoriella miculiformis): 912. coccinatum, Trypethelium (= Pyrenula cruenta): 978.

coccineum, Astrothelium: 798(k), 824(f), 854, SX(t). collospora, Bogoriella: 903(k), 906(f), 908, 988, SX(t). collospora, Mycomicrothelia (=Bogoriella collospora): 908 . collospora, Pyrenula (=Bogoriella collospora): 908.

colombiense, Astrothelium: 612(f), 613, 649, 814(k), 843(f), SX(t)

columbianum, Architrypethelium: 790(f), 792(k), 793, $\mathrm{SX}(\mathrm{t})$.

columbianum, Trypethelium (=Architrypethelium columbianum): 793.

columellata, Laurera (=Astrothelium meristosporum): 841(f), 871.

comparatula, Arthopyrenia (= Polymeridium quinqueseptatum): 949

composita, Pseudopyrenula (=Nigrovothelium tropicum): 931. compositum, Bathelium (=Nigrovothelium tropicum): 931. compositum, Trypethelium (=Nigrovothelium tropicum): 931 . compunctum, Leucodecton: 978.

concamerata, Porina (=Constrictolumina cinchonae): 919. concamerata, Verrucaria (= Constrictolumina cinchonae): 919. concatervata, Pseudopyrenula (=Viridothelium virens): 975. concatervata, Verrucaria (=Viridothelium virens): 975.

concatervatum, Trypethelium (=Viridothelium virens): 975 . condoricum, Astrothelium: 612(f), 614, 812(k), 842(f), $\mathrm{SX}(\mathrm{t})$.

confluens (Müll. Arg.) Stizenb., Verrucaria (=Bogoriella confluens): 909.

confluens (Weber) Hoffm., Verrucaria (=Bogoriella confluens): 909.

confluens, Arthopyrenia (=Constrictolumina leucostoma): 920 . confluens, Astrothelium: $818(\mathrm{k}), 847(\mathrm{f}), 855, \mathrm{SX}(\mathrm{t})$. confluens, Bogoriella: 905(k), 907(f), 909, SX(t). confluens, Cryptothelium (=Astrothelium confluens): 855. confluens, Heufleria (=Astrothelium confluens): 855. confluens, Microthelia (=Bogoriella confluens): 909. confluens, Mycomicrothelia (=Bogoriella confluens): 909. confluens, Pseudopyrenula (=Pseudopyrenula subnudata): 960. confusum, Astrothelium (=Astrothelium crassum): 782, 839(f), 856.

conglobatum, Trypethelium (=non-lichenized fungus): 978.

congregans, Astrothelium (=Anthracothecium australiense): 977.

conica, Heufleria (=Astrothelium cinnamomeum): 795, 854.

conica, Pseudopyrenula (= Saccardoella sp.): 977.

conicum var. pallidum, Astrothelium (= Astrothelium macrocarpum): 867.

conicum, Astrothelium (=Astrothelium cinnamomeum): 619, 628, 782, 795, 854 .

conigerum, Astrothelium (=Astrothelium robustum): 883 .

connivens Nyl., Trypethelium (=Astrothelium sepultum): 846(f), 887.

connivens Stirt., Trypethelium (=Pyrenula cruenta): 978. connivens, Bathelium (=Astrothelium sepultum): 887 . connivens, Laurera (=Astrothelium sepultum): 887.

conothelena var. errans, Microthelia (=Bogoriella conothelena): 909.

conothelena var. errans, Verrucaria (=Bogoriella conothelena): 909. conothelena, Bogoriella: 904(k), 905(k), 907(f), 909, $\mathrm{SX}(\mathrm{t})$.

conothelena, Microthelia (=Bogoriella conothelena): 909 . conothelena, Mycomicrothelia (=Bogoriella conothelena): 909.

conothelena, Verrucaria (=Bogoriella conothelena): 909. consimile, Astrothelium: 817(k), 846(f), 855, SX(t). consimile, Cryptothelium (=Astrothelium consimile): 855 . consimilis, Heufleria (=Astrothelium consimile): 855.

Constrictolumina: $681,683,748,749(\mathrm{p}), 751,756$, 756, 758, 769, 778, 786, 788(k), 917, 933, 953.

contendens, Arthopyrenia (= Polymeridium contendens): 945.

contendens, Polymeridium: 929(f), 933, 935(k), 945, $\mathrm{SX}(\mathrm{t})$.

contendens, Verrucaria (= Polymeridium contendens): 945.

corallinum, Astrothelium: 612(f), 614, 703, 705, 816(k), 846(f), SX(t).

corticatum, Polymeridium: 936(k), 940(f), 945, $\mathrm{SX}(\mathrm{t})$.

costaricense, Polymeridium: 937(k), 941(f), 945, SX(t). crassum var. tuberculosum, Trypethelium (=Astrothelium tuberculosum): 893.

crassum, Astrothelium: 655, 729(t), 730(p), 743(t), 754(p), 783(t), 809(k), 839(f), 855, 877, 891, SA(a), $\mathrm{SX}(\mathrm{t})$.

crassum, Pyrenodium (=Astrothelium crassum): 855 .

crassum, Trypethelium (=Astrothelium crassum): 855 .

croceum, Astrothelium: 668, 671, 743(t), 752(p), 780(f), 781, 808(k), 837(f), 856, SA(a), $S B(\mathrm{p}), S C(\mathrm{p}), \mathrm{SX}(\mathrm{t})$. croceum, Spermatodium (=Astrothelium aeneum): 821 . crocosarca, Trypethelium (= Trypethelium eluteriae): 964 .

cruenta, Pyrenula: 978.

cruentatum, Trypethelium (=Pyrenula cruenta): 978. cruentulum, Trypethelium (= Pyrenula cruenta): 978. cruentum var. subdecolor, Trypethelium (=Pyrenula cruenta): 978.

cruentum, Trypethelium (= Pyrenula cruenta): 978.

cryptolucens, Astrothelium: 644, 644, 645(f), 817(k), 846(f), SX(t).

cryptotheca, Pseudopyrenula: 953(k), 955, SX(t).

Cryptothecia: 977.

cryptothelia, Pyrenula: 977.

Cryptothelium (=Astrothelium ): 640, 651, 741, 749, 755, 781, 782, 792, 795, 796(k).

cryptothelium, Astrothelium (= Pyrenula cryptothelia): 977. cubana, Pseudopyrenula: 954(k), 955, 957(f), SX(t). cubanum, Plagiotrema (= Pseudopyrenula cubana): 953, 955. cumingiana, Melanotheca (= Marcelaria cumingii): 930.

cumingianum, Trypethelium (= Marcelaria cumingii): 930. cumingii, Bathelium (= Marcelaria cumingii): 930.

cumingii, Laurera (=Marcelaria cumingii): 930.

cumingii, Marcelaria: 746(t), 750(p), 780(f), 781, 928(k), 929(f), 930, SA(a), SX(t)

cumingii, Trypethelium (=Marcelaria cumingii): 930.

curvatum, Astrothelium: 715(f), 715, 721, 722(f), 818(k), 848(f), SX(t).

curvisporum, Astrothelium: 631, 695(f), 696, 708(f), 803(k), 831(f), SX(t).

dealbens, Polyblastiopsis (= Dictyomeridium amylosporum): 923.

decemseptatum, Astrothelium: 695(f), 696, 708(f), $800(\mathrm{k}), 827(\mathrm{f}), \mathrm{SX}(\mathrm{t})$.

decipiens, Anthracothecium (=Bogoriella decipiens): 909. 
decipiens, Bogoriella: 779(f), 902, 905(k), 907(f), 909, $\mathrm{SX}(\mathrm{t})$.

decipiens, Mycomicrothelia (= Bogoriella decipiens): 909. decolorata, Verrucaria (=Astrothelium phlyctaena): 877. deforme Fée, Trypethelium (=Astrothelium deforme): 795, 856, 975.

deforme Makhija \& Patw., Trypethelium (=Viridothelium indutum): 975.

deforme, Astrothelium: 813(k), 842(f), 856, SX(t). deforme, Bathelium (=Astrothelium deforme): 856 .

deformis Fée, Trypethelium (=Viridothelium indutum): 975.

defossa, Heufleria (=Astrothelium defossum): 857.

defossum, Astrothelium: 817(k), 847(f), 856, SX(t). defossum, Campylothelium: 977.

defossum, Cryptothelium (=Astrothelium defossum): 857.

degenerans, Astrothelium: 743(t), 753, 754(p), 755, 780(f), 785, 799(k), 826(f), 857, 864, 895, SA(a), $\mathrm{SC}(\mathrm{p}), \mathrm{SX}(\mathrm{t})$.

degenerans, Bathelium (=Astrothelium degenerans): 857.

degenerans, Pseudopyrenula (=Astrothelium degenerans): 857. degenerans, Trypethelium (=Astrothelium degenerans): 857 deightonii, Pseudopyrenula (=Nigrovothelium tropicum): 931. dermatodes, Pyrenula: 977.

detincta, Didymosphaeria (=Bogoriella miculiformis): 912 .

deustum, Trypethelium (=Botryosphaeria sp.): 976.

dichroum, Trypethelium (=Astrothelium scoria): 885.

dicoloratum, Astrothelium: 614, 616(f), 804(k), 832(f), $\mathrm{SX}(\mathrm{t})$.

Dictyomeridium: $748,749(\mathrm{p}), 749,751,753, \mathbf{7 5 6}$, 757, 769, 778, 788(k), 922, 934, 953.

diffluens, Pseudopyrenula (=Astrothelium pulcherrimum): 881 .

diffluens, Verrucaria (=Astrothelium pulcherrimum): 823(f), 881.

diluta var. degenerans, Pseudopyrenula (= Pseudopyrenula subnudata): 957(f), 960.

diluta, Pseudopyrenula: 635, 688(t), 701, 747(t), 750(p), 775, 776(f), 779(f), 785, 953, 954(k), 956, 957(f), 958, 961, SA(a), SX(t).

diluta, Pyrenula (= Pseudopyrenula diluta): 956.

diluta, Verrucaria (= Pseudopyrenula diluta): 956.

diphloea, Clathroporina (=Astrothelium megaspermum): 870.

diplocarpa, Heufleria (=Astrothelium diplocarpum): 857.

diplocarpoides, Astrothelium: 631, 641, 648, 733, 779(f), 807(k), 835(f), 857, 857, SX(t).

diplocarpum, Astrothelium: 671, 731, 732, 733, 743(t), 754(p), 807(k), 818(k), 836(f), 847(f), 857, SA(a), $\mathrm{SB}(\mathrm{p}), \mathrm{SX}(\mathrm{t})$.

diplocarpum, Cryptothelium (=Astrothelium diplocarpum): 857 . diremta, Pseudopyrenula (= Pseudopyrenula diluta): 956.

diremta, Verrucaria (= Pseudopyrenula diluta): 956.

discolor, Trypethelium (=Astrothelium macrocarpum): 832(f), 868.

disjunctum, Astrothelium: 695(f), 697, 711, 801(k), $830(\mathrm{f}), \mathrm{SX}(\mathrm{t})$.

dissimilum, Astrothelium: 799(k), 827(f), 858, SX(t). dissimilum, Trypethelium (=Astrothelium dissimilum): 858 . dissimulum, Astrothelium: 627, 827(f).

Distothelia: 751, 769, 778, 786, 787(k), 912, 926.

dithecium, Polymeridium: 936(k), 940(f), 945, SX(t). dodgei, Laurera (=Astrothelium subdisjunctum): 845(f), 890 .

dubia, Pseudopyrenula: 688(t), 774(f), 954(k), 957(f), 958, $\mathrm{SX}(\mathrm{t})$ duplex f. simplicius, Bathelium (=Astrothelium pulcherrimum): 881

duplex f. simplicius, Trypethelium (=Astrothelium pulcherrimum): 881.

duplex var. simplicior, Pseudopyrenula (=Astrothelium pulcherrimum): 881.

duplex, Bathelium (=Astrothelium phlyctaena): 877 .

duplex, Pseudopyrenula (=Astrothelium phlyctaena): 877 .

duplex, Trypethelium (=Astrothelium phlyctaena): 877.

duplicatum, Astrothelium: 695(f), 697, 705, 812(k), 842(f), SX(t).

ecuadoriense, Astrothelium: 615, 616(f), 673, 674, 819(k), 848(f), SX(t).

effusa, Laurera (=Astrothelium effusum): 858.

effusum, Astrothelium: 671, 687(t), 814(k), 858, SX(t).

elatior, Aptrootia: 743(t), 752(p), 771(f), 789(k), 789, 790(f), SA(a), SX(t).

elatior, Laurera (=Aptrootia elatior): 789.

elatius, Ascidium (= Aptrootia elatior): 789 .

elatius, Leptotrema (=Aptrootia elatior): 789.

elatius, Thelotrema (=Aptrootia elatior): 789 .

elegans, Laurera (=Bathelium mastoideum): 900 .

elegans, Thelenella (=Bathelium mastoideum): 900 .

elixii, Astrothelium: 663(f), 664(f), 667, 687(t), 811(k), 841(f), SX(t).

elliptica, Pseudopyrenula (=Pseudopyrenula subnudata): 960 .

elmeri, Pseudopyrenula (=Astrothelium heterophorum): 840(f), 862.

elmeri, Trypethelium (=Astrothelium heterophorum): 862 .

eluteriae ssp. subsulphurea, Pseudopyrenula (= Trypethelium eluteriae): 964, 966(f).

eluteriae var. anacardii, Pseudopyrenula (= Trypethelium eluteriae): 964.

eluteriae var. anacardii, Trypethelium (= Trypethelium eluteriae): 964.

eluteriae var. citrinum, Trypethelium (= Trypethelium eluteriae): 964.

eluteriae var. conglobatum, Trypethelium (= non-lichenized fungus): 978 .

eluteriae var. endochlorum, Trypethelium (= Trypethelium eluteriae): 964

eluteriae var. expallidum, Trypethelium (= Trypethelium eluteriae): 964.

eluteriae var. inaequale, Trypethelium (=Trypethelium inaequale): 969.

eluteriae var. nigricans, Trypethelium (= Trypethelium eluteriae): 964.

eluteriae var. polystomum, Trypethelium (= Trypethelium subeluteriae): 967(f), 972.

eluteriae var. sprengelii, Pseudopyrenula (= Trypethelium eluteriae): 964.

eluteriae var. sprengelii, Trypethelium (= Trypethelium eluteriae): 964.

eluteriae var. subsulphureum, Trypethelium (= Trypethelium eluteriae): 964.

eluteriae var. truncatum, Trypethelium (=Trypethelium eluteriae): 964.

eluteriae var. virens, Trypethelium (= Viridothelium virens): 759 , 975.

eluteriae, Pseudopyrenula (= Trypethelium eluteriae): 964 .

eluteriae, Trypethelium: 689(t), 741, 747(t), 750(p), 753, 755, 766, 772(f), 775, 776(f), 777(f), 780(f), 
781, 784, 961, 962, 963(k), 964, 966(f), 966, 967, 989, SA(a), $S B(\mathrm{p}), S C(\mathrm{p}), S X(\mathrm{t})$.

endochraceum, Trypethelium (=Astrothelium endochryseum): 827(f).

endochrysea, Pseudopyrenula (=Astrothelium endochryseum): 858.

endochryseum, Astrothelium: 646, 653, 654, 743(t), 752(p), 799(k), 826(f), 858, 895, SA(a), SX(t).

endochryseum, Bathelium (=Astrothelium endochryseum): 858. endochryseum, Trypethelium (=Astrothelium endochryseum): 858.

endocrocinum, Polymeridium: 929(f), 935(k), 946, $\mathrm{SX}(\mathrm{t})$.

endoflavens, Polymeridium: 721, 722(f), 936(k), 939(f), $\mathrm{SX}(\mathrm{t})$.

endosulphureum, Trypethelium (=Astrothelium luridum): 827(f), 867.

endoxantha, Pseudopyrenula: 954(k), 956(f), 958, SX(t). endoxanthoides, Pseudopyrenula: $747(\mathrm{t}), 750(\mathrm{p})$, 755, 954(k), 958, 960, SA(a), $S B(\mathrm{p})$.

epapillata, Pyrenula (=Astrothelium variolosum): 894 .

epapillatum, Spermatodium (=Astrothelium variolosum): 894 . epileucodes, Trypethelium: 964(k), 967(f), 969, SX(t). epiphylla, Trypetheliopsis: 977.

epiphyllum, Astrothelium (= Trypetheliopsis epiphylla): 977. epiphyllum, Bathelium (= Phyllobathelium epiphyllum): 977.

epiphyllum, Phyllobathelium: 977.

ernstianum, Parathelium (=Architrypethelium nitens): 794 . ernstianum, Pleurothelium (=Architrypethelium nitens): 790(f), 794.

erubescens, Astrothelium: 687(t), 743(t), 752(p), SA(a), $S B(\mathrm{p}), S C(\mathrm{p})$.

erubescens, Trypethelium (=Astrothelium porosum): 879 .

erumpens Fée, Trypethelium (= Pyrenula): 978.

erumpens Stirt., Trypethelium (= Trypethelium erumpens): 978.

erumpens, Pseudopyrenula (= Pseudopyrenula diluta): 957.

esenbeckiana, Constrictolumina: $918(\mathrm{k}), 919(\mathrm{f}), \mathbf{9 2 0}$, $\mathrm{SX}(\mathrm{t})$.

esenbeckiana, Melanotheca (=Constrictolumina esenbeckiana): 920.

esenbeckiana, Tomasellia (=Constrictolumina esenbeckiana): 920.

eumultiseptatum, Astrothelium: 695(f), 698, 703, 705, 708(f), 807(k), 836(f), SX(t).

euporum, Trypethelium (=Astrothelium phlyctaena): 823(f), 878.

eustomum, Astrothelium: 687(t), 698, 717, 718, 727, 732, 733, 734, 772(f), 785, 806(k), 835(f), 859, SX(t). eustomum, Pyrenastrum (= Astrothelium eustomum): 859 .

eustomurale, Astrothelium: 621, 698, 700(f), 708(f), $718,817(\mathrm{k}), 846(\mathrm{f}), \mathrm{SX}(\mathrm{t})$.

euthelia, Verrucaria (=Astrothelium megaspermum): 844(f), 870.

euthelium, Astrothelium: 743(t), 754(p), SA(a), $S C(\mathrm{p})$. exasperata, Verrucaria (=Astrothelium annulare): 833, 836. exasperatum, Trypethelium (=Astrothelium annulare): 834 . exigua, Bogoriella: 904(k), 906(f), 910, SX(t).

exigua, Microthelia (=Bogoriella exigua): 910.

exigua, Mycomicrothelia (=Bogoriella exigua): 910 .

Exiliseptum (= Polymeridium): 742, 933.

exocanthum, Trypethelium (=Viridothelium virens): 974(f), 975. exostemmatis, Astrothelium: 816(k), 845(f), 859, SX(t). exostemmatis, Bathelium (=Astrothelium exostemmatis): 859 . exostemmatis, Laurera (=Astrothelium exostemmatis): 859 . fallaciosa, Julella: 746(t), 748, $750(\mathrm{p}), 751$, SA.

fallax, Astrothelium: 774(f), 809(k), 839(f), 859, SX(t). favulosum, Trypethelium: 976.

feeana, Pertusaria: 978.

feei, Astrothelium: 785, 799(k), 826(f), 860, 864, SX(t). feei, Bathelium (=Astrothelium feei): 860 .

feei, Chooicia (=Astrothelium phlyctaena): 877.

feei, Trypethelium (=Astrothelium feei): 860.

ferrugineum var. inornatum, Trypethelium (=Astrothelium ferrugineum): 836(f), 860 .

ferrugineum, Astrothelium: 628, 807(k), 836(f), 860, $\mathrm{SX}(\mathrm{t})$.

ferrugineum, Trypethelium (=Astrothelium ferrugineum): 860 .

fijiense, Astrothelium: 644, 645(f), 774(f), 796(k), 822(f), $\mathrm{SX}(\mathrm{t})$.

flavicans, Microthelia (=Bogoriella captiosa): 908.

flavicans, Pseudopyrenula (=Pseudopyrenula subnudata): 960.

flavoalbum, Trypethelium (=Astrothelium meiophorum): 829(f), 870.

flavocinereum, Trypethelium (=Astrothelium cinereorosellum): 822(f), 853 .

flavocoronatum, Astrothelium: 728, 729(t), 730(p), 731, 731, 732(f), 804(k), 832(f), SX(t).

flavoduplex, Astrothelium: 698, 699, 700(f), 702, 705, 708(f), 811(k), 841(f), SX(t).

flavomaculatum, Astrothelium: 615, 616(f), 717, 812(k), 842(f), SX(t).

flavomeristosporum, Astrothelium: 616(f), 617, 814(k), 844(f), SX(t).

flavomurisporum, Astrothelium: 700(f), 701, 708(f), 821(k), 849(f), SX(t).

flavoreagens, Pseudopyrenula: 688(t), 711, 953(k), 956(f), SX(t).

flavostiolatum, Astrothelium: 616(f), 617, 815(k), 844(f), SX(t).

flavostiolatum, Bathelium: 675(f), 676(f), 678, 688(t), 895(k), 897(f), SX(t).

flavostromatum, Astrothelium: $700(\mathrm{f}), 701,708(\mathrm{f})$, 799(k), 822, 825(f), SX(t).

flavosuperans, Pseudopyrenula: 682(f), 684(f), 685, 689(t), 953(k), 956(f), SX(t).

flavothecium, Polymeridium: 723, 936(k), 940(f), 946, $S X(t)$.

flavum, Astrothelium: 700(f), 701, 708(f), 798(k), 823, 825(f), SX(t).

floridanum, Astrothelium: 743(t), 744(t), 754(p), 772(f), 801(k), 830(f), 860, 869, SA(a), SC(p), SX(t).

floridanum, Trypethelium (=Astrothelium floridanum): 860 .

follmannii, Pseudopyrenula (=Polymeridium subcinereum): 951.

formosa, Laurera (= nom. nud.): 884.

formosanum, Trypethelium (=Astrothelium bicolor): 848 .

foveolatum, Trypethelium: $747(\mathrm{t}), 750(\mathrm{p}), 964(\mathrm{k})$, 968(f), 969, SA(a), SX(t).

fugax, Astrothelium (= Lithothelium fugax): 977.

fugax, Lithothelium: 977.

fulva, Clathroporina (=Astrothelium megaspermum): 870 .

fulva, Polyblastiopsis (=Astrothelium megaspermum): 870.

fulva, Thelenella (=Astrothelium megaspermum): 844(f), 870 . 
fumosula, Bogoriella: 904(k), 906(f), 910, SX(t). fumosula, Microthelia (=Bogoriella fumosula): 910 . fumosula, Mycomicrothelia (=Bogoriella fumosula): 910 . fusca, Melanotheca (=Pyrenula): 978.

fuscum, Trypethelium (= Pyrenula sp.): 978.

fusispora, Laurera (=Astrothelium subdiscretum): 890. galactina, Pseudopyrenula (= Pyrenula dermatodes): 977. galbineum, Astrothelium (=Astrothelium macrocarpum): 620, 727, 731, 732, 867.

galligenum, Astrothelium: 802(k), 831(f), 861, SX(t). galligenum, Trypethelium (=Astrothelium galligenum): 861 gaudichaldii, Pyrenula (=Nigrovothelium tropicum): 931. gaudichaldii, Verrucaria (=Nigrovothelium tropicum): 931. gigantea, Didymella (=Didymella gigantea): $918(\mathrm{f}), 921$. gigantospora, Laurera (=Astrothelium gigantosporum): 861 . gigantosporum, Astrothelium: 649, 650, 744(t), 754(p), 815(k), 845(f), 861, SA(a), SX(t).

gigantosporum, Bathelium (=Astrothelium gigantosporum): 861.

gigasporum, Astrothelium: 773(f), 811(k), 840(f), 861, $\mathrm{SX}(\mathrm{t})$.

glaucoatra, Arthopyrenia (=Polymeridium glaucoatrum): 946. glaucoatrum, Polymeridium: 929(f), 935(k), 946, SX(t). globolucidum, Trypethelium (= Trypethelium regnellii): $724,971$. globosum, Astrothelium: 715(f), 716, 720, 809(k), 839(f), SX(t).

grande, Architrypethelium: 769(f), 775, 776(f), 790(f), 792(k), 793, SX(t).

grande, Ascidium (=Architrypethelium grande): 793 .

grande, Phaeotrema (=Architrypethelium grande): 793.

graphicum, Astrothelium: 615, 617, 715(f), 716, 812(k), 842(f), SX(t).

Graphis: 756.

gregale, Anthracothecium: 978.

gregale, Trypethelium (=Anthracothecium gregale): 978 .

grossoides, Astrothelium: 783(t), 797(k), 822(f), 862, $\mathrm{SX}(\mathrm{t})$.

grossum, Astrothelium: 744(t), 752(p), 804(k), 833(f), 862, 862, SA(a), $S B(\mathrm{p}), S C(\mathrm{p}), \mathrm{SX}(\mathrm{t})$.

grossum, Trypethelium (=Astrothelium grossoides): $822(\mathrm{f}), 862$. guianense, Astrothelium: 616(f), 618, 819(k), 849(f), $\mathrm{SX}(\mathrm{t})$.

guianensis, Pseudopyrenula: 635, 954(k), SX(t).

hagmannii, Pyrenula (= Pseudopyrenula subnudata): 960 .

hebetum, Trypethelium (=Astrothelium nitidiusculum): 873 .

hemisphaerica, Bogoriella: 746(t), 750(p), 785, 905(k), 907(f), 910, SA(a), SX(t).

hemisphaerica, Microthelia (=Bogoriella hemisphaerica): 910.

hemisphaerica, Mycomicrothelia (=Bogoriella hemisphaerica): 910.

hemisphaerica, Porina: 978.

hemisphaericum, Trypethelium (= Porina hemisphaerica): 978. heterochroa, Pseudopyrenula (=Astrothelium aeneum): 821 . heterochroa, Pyrenula (=Astrothelium aeneum): 821 . heterochroa, Segestria (=Astrothelium aeneum): 821 . heterochroa, Verrucaria (=Astrothelium aeneum): 821, 825(f). heterophorum, Astrothelium: $810(\mathrm{k}), 840(\mathrm{f}), 862, \mathrm{SX}(\mathrm{t})$. Heufleria (=Astrothelium ): 795.

hexamera, Pseudopyrenula: 635, 954(k), SX(t).

Holstiella (= Trypethelium ): 961.

hyalinum, Architrypethelium: 641, 662, 665, 790(f), 792(k), 793, 793, SX(t). hypoxylon var macrocarpon, Astrothelium (=Astrothelium macrocarpum): 867.

hypoxylon, Astrothelium (=Astrothelium pyrenastrosulphureum): 883.

hypoxylon, Pyrenodium (=Astrothelium pyrenastrosulphureum): 883.

illota, Pseudopyrenula (= Lithothelium illotum): 977.

illotum, Lithothelium: 977.

immersum, Dictyomeridium: 923(k), 924(f), 925, SX(t). immersum, Polymeridium (= Dictyomeridium immersum): 925.

inaequale, Trypethelium: 962, 963(k), 967(f), 969, 972, SX(t).

inamoenum, Trypethelium (= Trypethelium inaequale): 747(t), 750(p), 967(f), 969, 972, SA.

inarense, Trypethelium (=Polycoccum sp.): 978.

inconspicua, Melanotheca (= Pyrenula ): 978.

inconspicuum, Trypethelium (=Pyrenula sp.): 978.

indica Makhija \& Patw., Laurera (=Astrothelium indicum): 863.

indica Upreti \& Ajay Singh, Laurera (=Astrothelium meristosporum): 841(f), 863, 871.

indicum, Astrothelium: 813(k), 843(f), 863, SX(t).

indicum, Trypethelium (=Astrothelium scoria): 885 .

indutum, Trypethelium (=Viridothelium indutum): 975.

indutum, Viridothelium: 637, 686, 802(k), 973(k), 974(f), 975, SX(t).

infernalis, Megalotremis: 621.

infossa, Pseudopyrenula (=Astrothelium infossum): 863.

infossa, Verrucaria (=Astrothelium infossum): 863.

infossum, Astrothelium: 687(t), $800(\mathrm{k}), 828(\mathrm{f}), 863$, $\mathrm{SX}(\mathrm{t})$.

infossum, Trypethelium (=Astrothelium infossum): 863 .

infraeluteriae, Trypethelium: 634(f), 636, 963(k), 966(f), SX(t).

infraleucotrypa, Pyrenula: 702, 703.

infuscatula var. tecomae, Pseudopyrenula (=Astrothelium scorioides): 830(f), 886.

infuscatula, Pseudopyrenula (=Astrothelium infuscatulum): 863. infuscatulum var. tecomae, Trypethelium (=Astrothelium scorioides): 886.

infuscatulum, Astrothelium: 774(f), $800(\mathrm{k}), 828(\mathrm{f})$, 863, $\mathrm{SX}(\mathrm{t})$.

infuscatulum, Trypethelium (=Astrothelium infuscatulum): 863.

insigne, Trypethelium (= Trypethelium eluteriae): 964, 966(f).

inspersaeneum, Astrothelium: 625, 643, 687(t), 755, 785, 798(k), 822, 824(f), 864, SX(t).

inspersoaeneum, Astrothelium: 744(t), 752(p), SA(a), $S B(\mathrm{p})$.

inspersogalbineum, Astrothelium: 618, 619(f), 804(k), 832(f), SX(t).

inspersomastoideum, Bathelium: 675(f), 676(f), 679, 688(t), 896(k), 898(f), SX(t).

inspersotuberculosum, Astrothelium: 663(f), 664(f), 668, 687(t), 802(k), 830(f), SX(t).

inspersum, Polymeridium: 688(t), 929(f), 935(k), 946, SX $(\mathrm{t})$.

inspersum, Viridothelium: 634(f), 636, 802(k), 973(k), 974(f), SX(t).

intercedens, Microthelia (=Bogoriella hemisphaerica): 910 .

interjectum, Astrothelium: 655, 772(f), 783(t), 805(k), 834(f), 864, SX(t). 
interlatens var. nudatum, Astrothelium (=Anthracothecium sp.): 977.

interlatens, Astrothelium (=Anthracothecium sp.): 977. intermedia, Microthelia (=Bogoriella exigua): 910.

intermedium, Astrothelium: 769(f), 772(f), 773(f), 810(k), 839(f), 864, SX(t).

interrupta, Clathroporina (=Astrothelium meristosporum): 871 . interrupta, Thelenella (=Astrothelium meristosporum): 871 . irregulare, Astrothelium: 815(k), 844(f), 865, SX(t). irregulare, Bathelium (=Astrothelium irregulare): 865 . irregularis, Clathroporina (=Astrothelium leioplacum): 866. irregularis, Laurera (=Astrothelium irregulare): 865 .

isabellina, Heufleria (=Astrothelium isabellinum): 865.

isabellinum Eschw., Astrothelium: 643, 818(k), 865, $865,977, \mathrm{SX}(\mathrm{t})$.

isabellinum Vain., Astrothelium (= Lithothelium obtectum): 977. isabellinum, Cryptothelium (=Astrothelium isabellinum): 865.

isohypocrellinum, Dictyomeridium: 781, 923(k), 924(f), 925, SX(t).

isohypocrellinum, Polymeridium (= Dictyomeridium isohypocrellinum): 925.

isthmospora, Distothelia: 924(f), 926, 927(k), 927, SX(t). javanica, Melanotheca (=Astrothelium meristosporum): 871 . javanicum, Meristosporum (=Astrothelium meristosporum): $795,871$.

jordanii, Polymeridium: 937(k), 946, SX(t).

jordanii, Pseudopyrenula (= Polymeridium jordanii): 946.

Julella: 741, 748, 749(p), 751.

julelloides, Polymeridium: 937(k), 941(f), 947, SX(t).

karnatakense, Trypethelium (=Viridothelium megaspermum): 975.

keralense, Astrothelium: 815(k), 865, SX(t).

keralensis, Laurera (=Astrothelium keralense): 865 .

kinabaluense, Viridothelium: 634(f), 637, 973(k), $802(\mathrm{k})$, 974(f), SX(t).

komposchii, Astrothelium: 619(f), 620, 815(k), 844(f), SX(t).

krempelhuberi, Trypethelium: 963(k), 965(f), 970, $\mathrm{SX}(\mathrm{t})$.

kundaraensis, Laurera (=Bathelium madreporiforme): 899 .

kunzei, Astrothelium: 744(t), 754(p), 755, 766(f), 780(f), 799(k), 822, 825(f), 865, SA(a), $S C(\mathrm{p}), \mathrm{SX}(\mathrm{t})$.

kunzei, Trypethelium (=Astrothelium kunzei): 865.

laevigatum, Astrothelium: 729(t), 730(p), 744(t), 754(p), 783(t), 805(k), 833(f), 866, SA(a), SB(p), SX(t).

laevithallinum, Astrothelium: 646, 647(f), 744(t), 752(p), 799(k), 826(f), SA(a), SX(t).

lageniferum, Plagiotrema: 976.

lageniferum, Trypethelium (=Astrothelium pustulatum): $883,976$.

lateralis, Bogoriella: 903(k), 906(f), 911, SX(t).

lateralis, Mycomicrothelia (=Bogoriella lateralis): 911 .

Laurera (=Astrothelium ): 640, 741, 749, 755, 765, 775, 782, 792, 795, 796(k).

laurerosphaerioides, Astrothelium: 619(f), 620, 813(k), 842(f), SX(t).

lauropaluanum, Architrypethelium: 641, 642(f), 743(t), $752(\mathrm{p}), 790(\mathrm{f}), 792(\mathrm{k}), \mathrm{SA}(\mathrm{a}), S B(\mathrm{p}), S C(\mathrm{p}), \mathrm{SX}(\mathrm{t})$.

Leightonia (=Astrothelium ): 795.

leioplaca, Clathroporina (=Astrothelium leioplacum): 866.

leioplacum, Astrothelium: 710, 820(k), 849(f), 866, SX(t). leprieurii, Phaeographis: 662. leprieurii, Trypethelium (=Astrothelium phlyctaena): 823(f), 878.

leprosulum, Trypethelium (= Trypethelium eluteriae): 964 .

leprosum, Trypethelium (=Astrothelium phlyctaena): 823(f), 878.

leptoseptatum, Viridothelium: 722(f), 724, 799(k), 972, 973(k), 974(f), SX(t).

leuckertii, Bogoriella: 903(k), 911, SX(t).

leuckertii, Mycomicrothelia (=Bogoriella leuckertii): 911.

leucoconicum, Astrothelium: 653, 729(t), 730(p), 744(t), 754(p), 806(k), 834(f), 866, SA(a), SX(t).

leucosessile, Astrothelium: 646, 647(f), 729(t), 730(p), 744(t)，752(p)，755，783(t)，797(k)，823(f)，SA(a), $S C(\mathrm{p}), \mathrm{SX}(\mathrm{t})$

leucostoma, Arthopyrenia (= Constrictolumina leucostoma): 920.

leucostoma, Constrictolumina: 917(k), 918(f), 920, $\mathrm{SX}(\mathrm{t})$.

leucostoma, Tomasellia (=Constrictolumina leucostoma): 920.

leucostomum (=Nyl.) C.W. Dodge, Trypethelium (= Trypethelium sphaerocephalum): 853, 971.

leucostomum Kremp., Trypethelium (=Astrothelium chrysostomum): 833(f), 853, 882, 971.

leucothallina, Microthelia (=Bogoriella thelena): 916.

leucothelium, Astrothelium: 653, 805(k), 833(f), 853, 866, $S X(t)$

leucotrypa, Pyrenula: 978.

leucotrypum, Trypethelium (=Pyrenula leucotrypa): 978.

lichenoidea, Zignoella (= Nigrovothelium tropicum): 931.

limitata, Pseudopyrenula (=Pseudopyrenula subnudata): 960.

lineare, Bathelium: 678, 746(t), 752(p), 813(k), 896(k), 898(f), 899, SA(a), SX(t).

linearis, Laurera (=Bathelium lineare): 899.

linearis, Polyblastiopsis (= Bathelium lineare): 899

longiflavens, Polymeridium: 723, 936(k), 939(f), SX(t).

longisporum, Astrothelium: 648, 649, 715(f), 717, $819(\mathrm{k}), 848(\mathrm{f}), \mathrm{SX}(\mathrm{t})$.

lucidomedullatum, Astrothelium: 619(f), 621, 816(k), 846(f), SX(t).

lucidostromum, Astrothelium: 619(f), 621, $817(\mathrm{k})$, 846(f), SX(t)

lucidothallinum, Astrothelium: 619(f), 621, $812(\mathrm{k})$, 841(f), SX(t).

lueckingii, Dictyomeridium: 682(f), 683, 684(f), 688(t), 923(k), 924(f), SX(t).

lugescens, Anthracothecium (=Astrothelium lugescens): 867. lugescens, Astrothelium: 819(k), 848(f), 867, SX(t).

lugescens, Campylothelium (=Astrothelium lugescens): 867. lugescens, Verrucaria (=Astrothelium lugescens): 867.

luridum, Astrothelium: 629, $800(\mathrm{k}), 827(\mathrm{f}), 867, \mathrm{SX}(\mathrm{t})$.

luridum, Trypethelium (=Astrothelium luridum): 867 .

luteolucidum, Trypethelium: 657, 686, 722(f), 724, 962(k), 965(f), SX(t).

luteonitens, Anthracothecium (=Architrypethelium uberinum): 791.

luteonitens, Verrucaria (=Aptrootia elatior): 790.

luteum, Trypethelium (= Trypethelium eluteriae): 964 .

lyrata, Arthopyrenia (=Constrictolumina lyrata): 921.

lyrata, Constrictolumina: $917(\mathrm{k}), 919(\mathrm{f}), \mathbf{9 2 1}, \mathrm{SX}(\mathrm{t})$. macrocarpa, Bogoriella: 903(k), 911, 912, SX(t).

macrocarpa, Mycomicrothelia (=Bogoriella macrocarpa): 911 . macrocarpa, Porina (=Astrothelium macrocarpum): 867. 
macrocarpa, Porophora (=Astrothelium macrocarpum): 867. macrocarpa, Pyrenula (=Astrothelium macrocarpum): 867. macrocarpon, Pyrenodium (=Astrothelium macrocarpum): 867. macrocarpum, Astrothelium: 618, 620, 673, 687(t), 729 (t), 730(p), 731, 732, 744(t), 754(p), 773(f), 774(f), 784, 804(k), 832(f), 867, SA(a), $S B(\mathrm{p}), S C(\mathrm{p}), S \mathrm{X}(\mathrm{t})$.

macrosporum, Astrothelium: 803(k), 814(k), 831(f), 843(f), 868, SX(t).

macrosporum, Trypethelium (=Astrothelium macrosporum): 868.

macrostiolatum, Astrothelium: 728, 729(t), 730(p), 731, 732(f), 732, 811(k), 840(f), SX(t).

macrostomoides, Astrothelium: 647(f), 648, 807(k), 835(f), SX(t).

macrostomum, Astrothelium: 648, 699, 715(f), 717, 807(k), 835(f), SX(t).

madreporiforme var. obscurior, Bathelium (= nonlichenized fungus): 977.

madreporiforme var. obscurior, Trypethelium (=nonlichenized fungus): 978 .

madreporiforme, Bathelium: $688(\mathrm{t}), 729(\mathrm{t}), 730(\mathrm{p})$, 746(t), 752(p), 785, 812(k), 895, 896(k), 898(f), 899, $\mathrm{SA}(\mathrm{a}), S C(\mathrm{p}), \mathrm{SX}(\mathrm{t})$.

madreporiforme, Trypethelium (=Bathelium madreporiforme): 899.

madreporiformis var. obscurior, Laurera (= non-lichenized fungus): 977.

madreporiformis var. pruinosa, Laurera (=Bathelium pruinolucens): 901.

madreporiformis, Laurera (=Bathelium madreporiforme): 899. magnoliae, Zignoella (= Nigrovothelium tropicum): 931 .

majuscula, Arthopyrenia (=Constrictolumina majuscula): 921.

majuscula, Constrictolumina: 769(f), 775, 776(f), 777(f), 779(f), 917(k), 918(f), 921, SX(t).

majuscula, Pseudopyrenula (= Pyrenula massariospora): 977.

majuscula, Verrucaria (=Constrictolumina majuscula): 921.

malaccitula, Arthopyrenia (=Constrictolumina malaccitula): 921 .

malaccitula, Constrictolumina: 746(t), 750(p), 779(f), 917(k), 918(f), 921, SA(a), SX(t).

malaccitula, Verrucaria (=Constrictolumina malaccitula): 921. mamillana, Pyrenula: 978.

Marcelaria: 741, 748, 749(p), 749, 753, 775, 787, 789(k), 928, 962.

marcida, Pyrenula (=Astrothelium marcidum): 868, 869.

marcida, Verrucaria (=Astrothelium marcidum): 868.

marcidum, Astrothelium: 773(f), 810(k), 839(f), 861, $868, \mathrm{SX}(\mathrm{t})$.

marcidum, Trypethelium (=Astrothelium marcidum): 868.

marginata, Laurera (= Bathelium mastoideum): 900.

marginatula, Pyrenula (=Pyrenula adacta): 976.

marginatum, Trypethelium (=Bathelium mastoideum): 898(f), 900.

massalongoi, Ocellularia: 976.

massariospora, Pyrenula: 977.

mastoideum var. convexum, Trypethelium (=Astrothelium bicolor): 847.

mastoideum var. macerum, Trypethelium (=Astrothelium feei): 826(f), 860 .

mastoideum, Bathelium: 679, 688(t), 772(f), 812(k), 895, 896(k), 898(f), 900, 900, SX(t).

mastoideum, Trypethelium (=Bathelium mastoideum): 900. media, Pseudopyrenula: 954(k).

medians, Trypethelium (= Trypethelium eluteriae): 964 .

mediocrassum, Astrothelium: 622, 623(f), 820(k), 849(f), SX(t).

megacrypticum, Astrothelium: 648, 650(f), 819(k), 848(f), SX(t).

megaeneum, Astrothelium: 668, 669(f), 670(f), 671, 687(t), 799(k), 823, 825(f), SX (t).

megaleia, Laurera (=Astrothelium megaleium): 869 .

megaleium, Astrothelium: 814(k), 843(f), 869, SX(t). megaleium, Bathelium (=Astrothelium megaleium): 869 .

megaleium, Trypethelium (=Astrothelium megaleium): 869 .

megalophthalmum, Astrothelium: 628, 641, 771(f), 803(k), 832(f), 869, SX(t).

megalophthalmum, Trypethelium (=Astrothelium megalophthalmum): 869.

megalospora, Parathelium (=Architrypethelium uberinum): 794.

megalospora, Verrucaria (=Architrypethelium uberinum): 794 .

megalostoma, Heufleria (=Astrothelium megalostomum): 869.

megalostomum, Astrothelium: 651, 666, 816(k), 846(f), $869, \mathrm{SX}(\mathrm{t})$.

megalostomum, Campylothelium (=Astrothelium megalostomum): 869.

megalostomum, Cryptothelium (=Astrothelium megalostomum): 869.

megapotamica, Thelenella (=Phyllobathelium chlorogastricum): 976.

megapotamicum, Phyllobathelium (=Phyllobathelium chlorogastricum): 976.

megasperma f. conica, Laurera (=Astrothelium megaspermum): 870 .

megasperma f. immersa, Laurera (=Astrothelium megaspermum): 844(f), 870 .

megasperma var. tasmanica, Laurera (=Pyrenula ravenelii): 977.

megasperma, Laurera (=Astrothelium megaspermum): 870 .

megaspermum var. tasmanicum, Bathelium (=Pyrenula ravenelii): 977.

megaspermum, Astrothelium: 611, 613, 628, 665, 666, 687(t), 717, 744(t), 752(p), 754(p), 755, 769(f), 774(f), 775, 776(f), 777(f), 779(f), 785, 815(k), 844(f), 870, 989, SA(a), $S B(\mathrm{p}), S C(\mathrm{p}), \mathrm{SX}(\mathrm{t})$.

megaspermum, Bathelium (=Astrothelium megaspermum): 870 . megaspermum, Pleurotrema (=Viridothelium megaspermum): 975

megaspermum, Trypethelium (=Astrothelium megaspermum): $870,975$.

megaspermum, Viridothelium: 811(k), 973(k), 974(f), 975, $\mathrm{SX}(\mathrm{t})$.

megaspora, Bogoriella: 769(f), 905(k), 907(f), 912, SX(t). megaspora, Mycomicrothelia (=Bogoriella megaspora): 912 . megatropicum, Astrothelium: 622, 623(f), 802(k), 830(f), SX(t).

megeustomum, Astrothelium: 718, 719(f), 774(f), $818(\mathrm{k}), 847(\mathrm{f}), \mathrm{SX}(\mathrm{t})$.

meghalayense, Trypethelium (=Astrothelium scoria): 885 .

megochroleucum, Astrothelium: 623(f), 624, 672, 797(k), 824(f), SX(t).

meiophorum, Astrothelium: 783(t), 801(k), 829(f), 870, $\mathrm{SX}(\mathrm{t})$.

meiophorum, Trypethelium (=Astrothelium meiophorum): 870. 
Meisneria DC.: 795.

Meissneria Fée (=Astrothelium ): 795.

Melanophloea: 742.

melanophthalma, Pyrenula: 978.

melanophthalmum f. arctecinctum, Trypethelium (=Pyrenula arctecincta): 978.

melanophthalmum, Trypethelium (=Pyrenula melanophthalma): 978.

melanospora, Bogoriella: $688(\mathrm{t})$.

melanosporum, Ascidium (=Aptrootia elatior): 790 .

melanothrix, Phyllothelium: 976.

melanothrix, Trypethelium: 976.

meristospermum, Astrothelium: 666, 667.

meristospora, Laurera (=Astrothelium meristosporum): 871 . meristosporoides, Astrothelium: 812(k), 841(f), 871, $\mathrm{SX}(\mathrm{t})$.

meristosporoides, Laurera (=Astrothelium meristosporoides): 871.

Meristosporum (=Astrothelium ): 795.

meristosporum, Astrothelium: 744(t), 754(p), 812(k), 841(f), 871, SA(a), SC(p), SX(t).

meristosporum, Bathelium (=Astrothelium meristosporum): 871.

meristosporum, Trypethelium (=Astrothelium meristosporum): 871.

mesoduplex, Astrothelium: 697, 698, 699, 700(f), 702, 705, 813(k), 842(f), SX(t).

microsporum, Polymeridium: 935(k), 939(f), 947, $\mathrm{SX}(\mathrm{t})$.

microsporum, Trypethelium (= Polymeridium microsporum): 947.

microstomum, Trypethelium (=Astrothelium cinereorosellum): 853.

microthelena, Verrucaria (=Bogoriella exigua): 910.

miculiformis var. detincta, Microthelia (=Bogoriella miculiformis): 912.

miculiformis var. detincta, Verrucaria (= Bogoriella miculiformis): 912.

miculiformis var. distincta, Verrucaria (=Bogoriella miculiformis): 912.

miculiformis, Bogoriella: 688(t), 746(t), 750(p), 904(k), 905(k), 907(f), 912, SA(a), SX(t).

miculiformis, Microthelia (=Bogoriella miculiformis): 912 .

miculiformis, Mycomicrothelia (=Bogoriella miculiformis): 912.

miculiformis, Verrucaria (=Bogoriella miculiformis): 912.

minus var. nigratum, Astrothelium (= Astrothelium nigratum): 872 .

minus, Astrothelium (=Astrothelium cinnamomeum): 854 .

minutissima, Arthopyrenia (=Pseudopyrenula subnudata): 960.

minutula, Bogoriella: 746(t), 750(p), 904(k), 906(f), 912, $\mathrm{SA}(\mathrm{a}), \mathrm{SX}(\mathrm{t})$.

minutula, Microthelia (=Bogoriella minutula): 912 .

minutula, Mycomicrothelia (=Bogoriella minutula): 912 .

mirabile, Bathelium: 675(f), 676(f), 678, 679, 679, 688(t), 896(k), 897(f), SX(t).

modesta, Bogoriella: 688(t), 769(f), 905(k), 907(f), 913, SX (t).

modesta, Microthelia (=Bogoriella modesta): 913 .

modesta, Mycomicrothelia (=Bogoriella modesta): 913.

monospora, fulella (=Aptrootia elatior): 790. monospora, Laurera (= Ocellularia massalongoi): 976. monosporum, Anthracothecium (=Aptrootia elatior): 790 . monosporum, Polyblastiopsis (=Aptrootia elatior): 790. montagnei, Trypethelium (= Trypethelium eluteriae): 964 . multiforme, Polymeridium: 688(t), 936(k), 940(f), 947, SX(t).

multiseptatum, Polymeridium: 936(k), 947, SX(t). muriformis, Ornatopyrenis (=Bogoriella decipiens): 909.

Mycomicrothelia: 741, 748, 751, 758, 767, 769, 778, 786, 902, 933.

myriocarpa, Pyrenula (=Astrothelium scoria): 828(f), 885 . myriocarpa, Verrucaria (=Astrothelium scoria): 885.

myriocarpum, Spermatodium (=Astrothelium scoria): 885 . myriocarpum, Trypethelium (=Astrothelium scoria): 885 . myriococca, Verrucaria (=Astrothelium aeneum): 821 . myriomma, Pseudopyrenula (=Astrothelium annulare): 834 . myriomma, Verrucaria (=Astrothelium annulare): 831(f), 834. Myriotrema: 977.

nanosporum, Lithothelium: 978.

nanosporum, Trypethelium (= Lithothelium nanosporum): 978.

neblinae, Polymeridium: 937(k), 941(f), 948, SX(t).

neglecta, Pseudopyrenula (=Astrothelium nitidiusculum): 829(f), 873.

neglectum, Astrothelium: 728, 729(t), 730(p), 731, 733, 734(f), 806(k), 835(f), SX(t).

neodiplocarpum, Astrothelium: 669(f), 670(f), 671, 687(t), 810(k), 840(f), SX(t).

neogalbineum, Astrothelium: 643, 744(t), 752(p), 772(f), 796(k), 822(f), 864, 872, SA(a), $S B(\mathrm{p}), S C(\mathrm{p})$, $\mathrm{SX}(\mathrm{t})$.

neogalbineum, Trypethelium (=Astrothelium neogalbineum): 872.

neoinspersum, Astrothelium: 623(f), 624, 744(t), 745(t), 752(p), 798(k), 822, 824(f), SA(a), SC(p), SX(t).

neovariolosum, Astrothelium: 728, 729(t), 730(p), 731, 734, 734, 735(f), 736, 783(t), 805(k), 833(f), SX(t).

neuwirthii, Polymeridium: 937(k), 940(f), 948, SX(t). nicaraguense, Astrothelium: 649, 650(f), 745(t), 754(p), $816(\mathrm{k}), 820(\mathrm{k}), 845(\mathrm{f}), 849(\mathrm{f}), \mathrm{SA}(\mathrm{a}), S C(\mathrm{p}), \mathrm{SX}(\mathrm{t})$.

nieteriana, Arthopyrenia (=Arthopyrenia nieteriana): 920 . nigeriensis, Laurera (=Astrothelium subdiscretum): 890.

nigratum, Astrothelium: 783(t), 801(k), 829(f), 872, $\mathrm{SX}(\mathrm{t})$.

nigricans, Astrothelium: 783(t), 809(k), 838(f), 872, $872, \mathrm{SX}(\mathrm{t})$.

nigritulum, Trypethelium (= Pyrenula ): 978.

nigrocacuminum, Astrothelium: 651, 653, 669(f), 670 (f), 672, 687(t), 806(k), 835(f), SX(t).

nigroporum, Bathelium: 779(f), $800(\mathrm{k}), 896(\mathrm{k}), 897(\mathrm{f})$, 900, SX(t).

nigroporum, Trypethelium (=Bathelium nigroporum): 900 . nigrorufum, Astrothelium: 799(k), 827(f), 873, SX(t). nigrorufum, Trypethelium (=Astrothelium nigrorufum): 873.

Nigrovothelium: 748, 749(p), 753, 755, 757, 788(k), 930. nigrum, Astrothelium: 702, 704(f), 783(t), 809(k), 838(f), $\mathrm{SX}(\mathrm{t})$.

nitens, Architrypethelium: 743(t), 752(p), 777(f), 779(f), 790(f), 792(k), 794, SA(a), SX(t).

nitens, Verrucaria (=Architrypethelium nitens): 794 .

nitidiuscula, Pseudopyrenula (=Astrothelium nitidiusculum): 873. 
nitidiuscula, Verrucaria (=Astrothelium nitidiusculum): 873. nitidiusculum, Astrothelium: 633, 654, 674, 745(t), 752(p), 755, 766(f), 771(f), 772(f), 773(f), 774(f), 783(t), 784, 801(k), 829(f), 850, 863, 873, 884, $\mathrm{SA}(\mathrm{a}), S C(\mathrm{p}), \mathrm{SX}(\mathrm{t})$.

nitidiusculum, Trypethelium (=Astrothelium nitidiusculum): 766, 782, 784, 850, 873, 885, 962.

nitidulum, Astrothelium: 803(k), 831(f), 874, SX(t). nitidum Müll. Arg., Campylothelium (= Dictyomeridium proponens): 870, 926.

nitidum Zahlbr., Campylothelium (=Astrothelium megaspermum): 870.

nobilis, Zignoella (= Nigrovothelium tropicum): 931.

nonensis, Bogoriella: 904(k), 905(k), 906(f), 913, SX(t). nonensis, Microthelia (=Bogoriella nonensis): 913. nonensis, Mycomicrothelia (=Bogoriella nonensis): 913. nonensis, Verrucaria (=Bogoriella nonensis): 913. norisianum, Astrothelium: 651, 652(f), 745(t), 752(p), 772(f), 817(k), 846(f), SA(a), SB(p),SC(p), SX(t).

novemseptatum, Astrothelium: 703, 704(f), 708(f), $810(\mathrm{k}), 840(\mathrm{f}), \mathrm{SX}(\mathrm{t})$.

Novomicrothelia: 748, 749(p), 751, 757, 758, 767, 769, 778, 786, 787(k), 912, 933, 953.

nudum, Trypethelium (=Pyrenula sp.): 978.

oahuensis, Pseudopyrenula (= Pseudopyrenula oahuensis): 958. oblitescens, Pyrenastrum (= Pyrenula cruenta): 978. oblitescens, Trypethelium (= Pyrenula cruenta): 978 . obovata, Bogoriella: 905(k), 908, 913, SX(t). obovata, Microthelia (=Bogoriella obovata): 913 . obovata, Mycomicrothelia (=Bogoriella obovata): 913. obovata, Pyrenula (=Bogoriella obovata): 913.

obovata, Verrucaria (=Bogoriella obovata): 913.

obscurum, Astrothelium: 745(t), 754(p), 783(t), 785, 809(k), 838(f), 874, SA(a), SC(p), SX(t).

obtectum, Astrothelium: 651, 652(f), 654, 729(t), 730(p), 745(t), 752(p), 806(k), 835(f), SA(a), SX(t).

obtectum, Lithothelium: 977.

obvelata, Arthopyrenia (=Polymeridium siamense): 950. obvoluta, Pseudopyrenula (= Pyrenula obvoluta): 977. obvoluta, Pyrenula: 977.

ocellata, Polyblastia (= Polymeridium ocellatum): 948. ocellata, Polyblastiopsis (= Polymeridium ocellatum): 948. ocellata, Verrucaria (= Polymeridium ocellatum): 948. ocellatum, Astrothelium: 808(k), 837(f), 874, SX(t). ocellatum, Exiliseptum (= Polymeridium ocellatum): 933, 948. ocellatum, Polymeridium: 933, 937(k), 948, SX(t). ocellatum, Trypethelium (= Pyrenula mamillana): 978. Ocellularia: 756, 976.

ochrocleistum, Astrothelium (= Pyrenula prostrata): 977.

ochroleuca var. effusa, Pseudopyrenula (= Astrothelium pulcherrimum): 881.

ochroleuca var. pallescens, Pseudopyrenula (=Astrothelium phlyctaena): 878.

ochroleuca var. subdissocians, Pseudopyrenula (=Astrothelium subdissocians): 891.

ochroleuca, Pseudopyrenula (=Astrothelium phlyctaena): 877. ochroleuca, Verrucaria (=Astrothelium phlyctaena): 823(f), 877. ochroleucodes, Laurera (=Cryptothecia sp.): 977.

ochroleucoides, Astrothelium: 614, 624, 674, 677, 679, 680, 681, 698, 703, 704(f), 812(k), 841(f), SX(t).

ochroleucum var. depauperatum, Trypethelium (=Astrothelium phlyctaena): 878 . ochroleucum var. effusum, Trypethelium (=Astrothelium pulcherrimum): 881.

ochroleucum var. erubescens, Trypethelium (=Astrothelium porosum): 879.

ochroleucum var. pallescens, Trypethelium (=Astrothelium phlyctaena): 877.

ochroleucum var. subdissocians, Trypethelium (=Astrothelium subdissocians): 891.

ochroleucum, Astrothelium: 755.

ochroleucum, Spermatodium (=Astrothelium phlyctaena): 877.

ochroleucum, Trypethelium (=Astrothelium phlyctaena): 648, 782, 784, 877, 878, 879, 881, 962.

ochrothelioides, Astrothelium (=Astrothelium macrocarpum): 832(f), 867.

ochrothelium, Astrothelium: 785, 804(k), 833(f), 874, $\mathrm{SX}(\mathrm{t})$.

ochrothelium, Trypethelium (=Astrothelium ochrothelium): 874.

ochrothelizum (Müll. Arg.) Nyl., Trypethelium (=Astrothelium macrocarpum): 832(f), 867.

ochrothelizum Nyl., Trypethelium (=Astrothelium macrocarpum): 867.

ochrothelizum, Astrothelium (=Astrothelium macrocarpum): $731,867$.

octomera, Pseudopyrenula (= Saccardoella sp.): 977.

octoseptatum, Astrothelium: 704(f), 705, 706, 708(f), 806(k), 835(f), SX(t).

octospora, Heufleria (=Astrothelium octosporum): 875 .

octospora, Laurera (=Astrothelium octosporoides): 875.

octosporoides, Astrothelium: 687(t), 816(k), 875, SX(t). octosporum, Astrothelium: 622, 817(k), 847(f), 875, $875, \mathrm{SX}(\mathrm{t})$.

octosporum, Bathelium (=Astrothelium octosporoides): 875 . octosporum, Cryptothelium (=Astrothelium octosporum): 875. oculata, Arthopyrenia (= Polymeridium sulphurescens): 952. oculatum, Polymeridium (= Polymeridium sulphurescens): 952. oleagina, Pyrenula: 978.

oleosa, Mycomicrothelia (=Novomicrothelia oleosa): 748, $758,933$.

oleosa, Novomicrothelia: $688(\mathrm{t}), 747(\mathrm{t}), 750(\mathrm{p}), \mathbf{7 5 8}$, 758, 777(f), 903(k), 906(f), 933, 933, SA(a), SX(t).

oligocarpum, Astrothelium: 783(t), 809(k), 838(f), $875, \mathrm{SX}(\mathrm{t})$.

oligocarpum, Trypethelium (=Astrothelium oligocarpum): 875 . oligospora, Laurera (=Astrothelium megaleium): 869 .

oligosporum, Bathelium (=Astrothelium megaleium): 869 . oligosporum, Trypethelium (=Astrothelium megaleium): 869.

olivaceofuscum, Astrothelium: 687(t), 803(k), 832(f), 876, SX $(\mathrm{t})$.

olivaceofuscum, Trypethelium (=Astrothelium olivaceofuscum): 876.

operculicola, Massarina (=Polymeridium subcinereum): 951. ornata, Melanotheca (= Pyrenula cruenta): 978.

Ornatopyrenis (= Ornatopyrenis ): 902.

ornatum (= Müll. Arg.) Hellb., Trypethelium (= Pyrenula cruenta): 970, 978, 978.

ornatum Müll. Arg., Trypethelium: 686, 964(k), 968(f), 970, 971, SX(t).

ostendata, Laurera (=Astrothelium megaspermum): 870 . ostendatum, Bathelium (=Astrothelium megaspermum): 870 . ostendatum, Trypethelium (=Astrothelium megaspermum): 844(f), 870. 
pachytheca, Bogoriella: 905(k), 914, SX(t).

pachytheca, Didymosphaeria (=Bogoriella pachytheca): 914 . pachytheca, Mycomicrothelia (=Bogoriella pachytheca): 914 . palaquii, Didymosphaeria (=Bogoriella thelena): 916.

pallescens Fée, Trypethelium (=Astrothelium phlyctaena): 859, 877.

pallescens Leight., Trypethelium (=Astrothelium fallax): 859 .

pallescens var. subdissocians, Trypethelium (=Astrothelium subdissocians): 891.

pallidoflavum, Astrothelium: 669(f), 670(f), 672, 673, 687(t), 799(k), 807(k), 825(f), 836(f), SX(t).

pallidum, Trypethelium (=Anthracothecium prasinum): 978 .

papillatum, Trypethelium (= Pyrenula quassiaecola): 978.

papillosa, Laurera (=Astrothelium papillosum): 876.

papillosa, Riddlea (=Bathelium madreporiforme): 895, 898(f), 899.

papillosum Ach., Trypethelium (=Astrothelium variolosum): 894, 969.

papillosum Knight, Trypethelium: 894.

papillosum Kremp., Trypethelium (= Trypethelium foveolatum): 969.

papillosum var. fuscum, Trypethelium (=Astrothelium variolosum): 894

papillosum, Astrothelium: 771(f), 815(k), 844(f), 876, $\mathrm{SX}(\mathrm{t})$.

papillosum, Bathelium (=Astrothelium variolosum): 894 .

papuana, Pseudopyrenula: 955(k), 956(f), 959, SX(t).

papulosa, Pseudopyrenula (=Astrothelium papulosum): 876.

papulosa, Verrucaria (=Astrothelium papulosum): 876.

papulosum, Astrothelium: 687(t), 801(k), 830(f), 876, $\mathrm{SX}(\mathrm{t})$.

papulosum, Trypethelium (=Astrothelium papulosum): 876 . paradoxum, Trypethelium (=Sclerophyton seriale): 978.

paraproponens, Dictyomeridium: 683, 923(k), 924(f), 926, $\mathrm{SX}(\mathrm{t})$.

paraproponens, Polymeridium (=Dictyomeridium paraproponens): 926.

parietina, Xanthoria: 662 .

parmularia, Astrothelium (=non-lichenized fungus): 977. parvicarpum, Trypethelium (=Astrothelium meiophorum): 829(f), 870, 871.

paulensis, Arthopyrenia (= Polymeridium pyrenuloides): 949.

pauperrima, Laurera (=non-lichenized fungus): 977.

pauperrimum, Trypethelium (=Astrothelium meiophorum): 978. Peltigera: 977.

peltigereum, Trypethelium (= Pyrenidium actinellum): 977.

pentameria, Pseudopyrenula (= Polymeridium albocinereum): 941(f), 942.

penuriixanthum, Architrypethelium: 662, 663(f), 664(f), 665, 687(t), 790(f), 792(k), 793, SX(t).

peranceps, Astrothelium: 783(t), 809(k), 838(f), 876, $\mathrm{SX}(\mathrm{t})$.

peranceps, Trypethelium (=Astrothelium peranceps): 876 .

perrotetii, Trypethelium (= Trypethelium eluteriae): 964 .

perspersum, Astrothelium: 623(f), 625, 745(t), 754(p), $755,800(\mathrm{k}), 828(\mathrm{f}), \mathrm{SA}(\mathrm{a}), \mathrm{SX}(\mathrm{t})$.

Pertusaria: 979.

pertusarina, Polyblastiopsis (=Astrothelium megaspermum): 870.

phaeomelodes, Bathelium (=Astrothelium subdiscretum): 843(f), 890 . phaeomelodes, Laurera (=Astrothelium subdiscretum): 890 . phaeomelodes, Trypethelium (=Astrothelium subdiscretum): 890.

phaeothelium, Astrothelium: $802(\mathrm{k}), \quad 830(\mathrm{f}), 877$, $\mathrm{SX}(\mathrm{t})$.

phaeothelium, Trypethelium (=Astrothelium phaeothelium): 877.

philippina, Didymosphaeria (=Bogoriella hemisphaerica): 910.

philippinense, Astrothelium: 623(f), 625, 814(k), 844(f), SX $(\mathrm{t})$.

phlyctaena var. pyrenuloides, Trypethelium (=Astrothelium phlyctaena): 878.

phlyctaena, Astrothelium: 646, 648, 687(t), 709, 745(t), 754(p), 780(f), 783(t), 784, 797(k), 823(f), 877, 879, $\mathrm{SA}(\mathrm{a}), S C(\mathrm{p}), \mathrm{SX}(\mathrm{t})$.

phlyctaena, Trypethelium (=Astrothelium phlyctaena): 877 . Phyllobathelium: 851.

pictum, Astrothelium: 641, 718, 719(f), 796(k), 822(f), $\mathrm{SX}(\mathrm{t})$.

Plagiotrema (=Pseudopyrenula ): 953

planior, Arthopyrenia (=Constrictolumina planorbis): 922.

planipes, Arthopyrenia (=Arthopyrenia planipes): 920.

planorbiculata, Arthopyrenia (=Constrictolumina planorbis): 922.

planorbis, Arthopyrenia (=Constrictolumina planorbis): 756, 922.

planorbis, Constrictolumina: 746(t), 750(p), 756, 917(k), 919(f), 922, SA(a), SC(p), SX(t).

planorbis, Verrucaria (=Constrictolumina planorbis): 756 , 922.

planum, Trypethelium (=Pyrenula subvariolosa): 978 .

platyleucostomum, Trypethelium: 747(t), 750(p), 971, $\mathrm{SA}(\mathrm{a}), S B(\mathrm{p}), S C(\mathrm{p})$.

platystomum f. leucostomum, Trypethelium (= Trypethelium sphaerocephalum): 968(f), 971.

platystomum var. denudatum, Trypethelium (= Trypethelium krempelhuberi): 965(f), 970.

platystomum, Trypethelium: $686,689(\mathrm{t}), 689,963(\mathrm{k})$, 965(f), 970, SX(t).

pleiomerella, Arthopyrenia (= Polymeridium pleiomerellum): 948.

pleiomerellum, Polymeridium: 785, 937(k), 940(f), 948, $\mathrm{SX}(\mathrm{t})$.

pleiomeroides, Arthopyrenia (= Polymeridium chioneum): 944 . pleiomeroides, Polymeridium (= Polymeridium chioneum): 944. pleiomeroides, Verrucaria (=Verrucaria pleiomeroides): 939(f).

pleiostomum, Astrothelium: $810(\mathrm{k}), 839(\mathrm{f}), 878, \mathrm{SX}(\mathrm{t})$.

pleurothecium, Polymeridium: 779(f), 937(k), 941(f), 949, SX $(\mathrm{t})$.

plicatorimosum, Trypethelium: 963(k), 967(f), 971, $\mathrm{SX}(\mathrm{t})$.

Polyblastiopsis: 751 .

polychroum, Trypethelium (=Astrothelium bicolor): 828(f), 848.

Polycoccum: 978

Polymeridium: 610, 714, 723(f), 723, 725, 741, 748, 749 (p), 749, 751, 753, 757, 766, 769, 775, 778, 788(k) 922, 931, 933, 953, 962, 987, 991.

Polymeridium, Arthopyrenia sect. (=Polymeridium ): 933.

polyphragmia, Pseudopyrenula (= Polymeridium quinqueseptatum): 949. 
Porina: 655

porinoides Ach., Verrucaria (=Astrothelium pupula): 882 . porinoides Mont., Verrucaria (=Astrothelium pupula): 882 . porinoides, Pseudopyrenula (=Astrothelium pupula): 824(f), 882.

porinosporum, Bathelium: $652(\mathrm{f}), 655,746(\mathrm{t}), 752(\mathrm{p})$, 774(f), 799(k), 800(k), 896(k), 897(f), SA(a), SB(p), $\mathrm{SC}(\mathrm{p}), \mathrm{SX}(\mathrm{t})$

porosa, Leightonia (=Astrothelium porosum): 795, 879 . porosa, Verrucaria (=Astrothelium porosum): 879.

porospora, Arthopyrenia (=Constrictolumina porospora): 922.

porospora, Constrictolumina: 917(k), 918(f), 922, SX(t). porosum, Astrothelium: 775, 776(f), 783(t), 784, 785, 797(k), 822(f), 879, 989, SX(t).

porosum, Bathelium (=Astrothelium porosum): 879 .

porosum, Trypethelium (=Astrothelium porosum): 879 .

portoricensis, Pseudopyrenula (=Astrothelium pulcherrimum): 881.

praetervisa var. cinerea, Heufleria (=Astrothelium cinereum): 854.

praetervisa, Heufleria (=Astrothelium praetervisum): 879 .

praetervisum var. cinereum, Cryptothelium (=Astrothelium cinereum): 854.

praetervisum, Astrothelium: 644, 818(k), 847(f), 854, 879, SX(t).

praetervisum, Cryptothelium (=Astrothelium praetervisum): 879.

prasinum, Anthracothecium: 978.

pringlei, Trypethelium (= Trypethelium eluteriae): 964, 966(f).

proponens, Campylothelium (= Dictyomeridium proponens): 757, 926.

proponens, Dictyomeridium: 683, 746(t), 750(p), 757, 757, 773(f), 785, 922, 923(k), 924(f), 926, SA(a), SX(t).

proponens, Polyblastia (=Dictyomeridium proponens): 757 , 926.

proponens, Polyblastiopsis (=Dictyomeridium proponens): 757, 926.

proponens, Polymeridium: 748, 749, 753, 757, 926.

proponens, Verrucaria (= Dictyomeridium proponens): 757 , 926.

prostans, Verrucaria (=Constrictolumina cinchonae): 919.

prostrata, Parmentaria (= Pyrenula prostrata): 977.

prostrata, Pyrenula: 977.

prostratum, Astrothelium (= Pyrenula prostrata): 977.

prostratum, Heufleridium (= Pyrenula prostrata): 977.

pruinolucens, Bathelium: $811(\mathrm{k}), 896(\mathrm{k}), 897(\mathrm{f}), 901$, $\mathrm{SX}(\mathrm{t})$.

pruinosum, Bathelium: 675(f), 676(f), 679, 680, 681, 688(t), 896(k), 897(f), SX(t).

pseudannulare, Astrothelium: 625, 626(f), 802(k), 808(k), 830(f), 837(f), SX(t).

pseudocyphellatum, Astrothelium: 783(t), 805(k), 834(f), 880, SX(t).

pseudodissimulum, Astrothelium: 626(f), 627, 799(k), 827(f), SX(t).

pseudoferrugineum, Astrothelium: 626(f), 627, 807(k), 836(f), SX(t)

pseudomegalophthalmum, Astrothelium: 626(f), 628, 803(k), 831(f), SX(t).

pseudoplatystomum, Astrothelium: $802(\mathrm{k}), \mathbf{8 8 0}, \mathrm{SX}(\mathrm{t})$. pseudoplatystomum, Trypethelium (=Astrothelium pseudoplatystomum): 880.
Pseudopyrenula: $613,635,653,723,741,748,749(\mathrm{p})$, 751, 755, 757, 767, 769, 770, 775, 777, 788(k), 953.

pseudovariata, Laurera (=Astrothelium pseudovariatum): 880.

pseudovariatum, Astrothelium: $813(\mathrm{k}), \mathbf{8 8 0}, \mathrm{SX}(\mathrm{t})$.

puiggarii var. pallescens, Campylothelium (=Astrothelium puiggarii): 880.

puiggarii, Astrothelium: 625, 627, 707, 716, 721, 771(f), 775, 776(f), 819(k), 848(f), 880, SX(t).

puiggarii, Campylothelium (=Astrothelium puiggarii): 658, $749,795,880$.

pulcherrima, Pseudopyrenula (=Astrothelium pulcherrimum): 881.

pulcherrimum, Astrothelium: 745(t), 754(p), 783(t), 784, 797(k), 823(f), 878, 879, 881, SA(a), SX(t).

pulcherrimum, Trypethelium (=Astrothelium pulcherrimum): 881.

punctata, Bogoriella: 688(t), 785, 903(k), 904(k), 906(f), 914, $\mathrm{SX}(\mathrm{t})$.

punctata, Mycomicrothelia (=Bogoriella punctata): 914.

punctulatum, Astrothelium: 783(t), 797(k), 822(f), $881, S X(t)$.

pupula, Astrothelium: 745(t), 754(p), 797(k), 824(f), 882, SA(a), SX(t).

pupula, Pseudopyrenula (=Astrothelium pupula): 882.

pupula, Pyrenula (=Astrothelium pupula): 882.

pupula, Trypethelium (=Astrothelium pupula): 882 .

pupuloides, Pseudopyrenula (=Astrothelium pupula): 882.

purpurascens, Astrothelium: 643, 644, 671, 745(t), 752(p), 781, 818(k), 847(f), 882, SA(a), SX(t).

purpurascens, Cryptothelium (=Astrothelium purpurascens): 882.

purpurascens, Heufleria (=Astrothelium purpurascens): 882 . purpurascens, Melanotheca (=Pyrenula cruenta): 978. purpurascens, Trypethelium (= Pyrenula cruenta): 978. purpurina, Laurera (= Marcelaria purpurina): 741, 930. purpurina, Marcelaria: $688(\mathrm{t}), 746(\mathrm{t}), 750(\mathrm{p}), 773(\mathrm{f})$, 775, 776(f), 780(f), 781, 785, 928(k), 928, 929(f), 930, SA(a), SX(t).

purpurinum, Bathelium (=Marcelaria purpurina): 930.

purpurinum, Trypethelium (=Marcelaria purpurina): 930. pustulata, Pseudopyrenula (=Astrothelium pustulatum): 882.

pustulatum, Astrothelium: 631, 803(k), 831(f), 882, $\mathrm{SX}(\mathrm{t})$.

pustulatum, Trypethelium (=Astrothelium pustulatum): 882 .

pustulosa, Verrucaria (= Polymeridium quinqueseptatum): 949. pyrenastraeum, Astrothelium (= Pyrenula septicollaris): 977.

pyrenastroides, Astrothelium (= Pyrenula pyrenastroides): 977.

italic $>$ pyrenastroides, Parmentaria (= Pyrenula pyrenastroides): 977.

pyrenastroides, Polymeridium: 932(f), 934(k), 949, $\mathrm{SX}(\mathrm{t})$.

pyrenastroides, Pyrenula: 977.

pyrenastroides, Verrucaria (= Pyrenula pyrenastroides): 977.

pyrenastrosulphureum, Astrothelium: 783(t), 808(k), $838(\mathrm{f}), \mathbf{8 8 3}, \mathrm{SX}(\mathrm{t})$.

Pyrenula: 638, 694, 703, 978.

pyrenuliforme, Astrothelium: 615, 669(f), 670(f), 673, $674,687(\mathrm{t}), 819(\mathrm{k}), 848(\mathrm{f}), \mathrm{SX}(\mathrm{t})$.

pyrenuloides, Arthopyrenia (= Polymeridium pyrenuloides): 949.

pyrenuloides, Bathelium (= Pyrenula pyrenuloides): 977 . 
pyrenuloides, Polymeridium: 932(f), 934(k), 949, SX(t). pyrenuloides, Pseudopyrenula (= Nigrovothelium tropicum): 931.

pyrenuloides, Pyrenula: 977, 978.

pyrenuloides, Trypethelium (= Pyrenula pyrenuloides): 978 . pyriformis, Laurera (=Bathelium mastoideum): 900 .

pyriformis, Polyblastiopsis (=Bathelium mastoideum): 900. quassiicola, Pyrenula: 978.

quassiicola, Trypethelium (=Astrothelium phlyctaena): 877. quatuorseptatum, Astrothelium: 704(f), 706, 708(f), $810(\mathrm{k}), 840(\mathrm{f}), \mathrm{SX}(\mathrm{t})$.

queenslandica, Bogoriella: 902, 905(k), 907(f), 914, $\mathrm{SX}(\mathrm{t})$.

queenslandica, Microthelia (=Bogoriella queenslandica): 914 .

queenslandica, Mycomicrothelia (=Bogoriella queenslandica): 914.

queenslandica, Ornatopyrenis (=Ornatopyrenis queenslandica): 902, 914.

quinqueseptata, Arthopyrenia (=Polymeridium quinqueseptatum): 949.

quinqueseptata, Verrucaria (= Polymeridium quinqueseptatum): 949.

quinqueseptatum, Polymeridium: 635, 688(t), 937(k), 940(f), 949, SX(t).

quintaria, Pseudopyrenula (=non-lichenized fungus): 977.

ramosii, Pseudopyrenula (= Myriotrema): 977.

ravenelii, Pyrenula: 977.

refertum, Plagiotrema (= Polymeridium refertum): 950.

refertum, Polymeridium: 937(k), 950, SX(t).

refertum, Trypethelium (=Polymeridium refertum): 950 .

regnellii, Trypethelium: 657, 724, 962(k), 965(f), 971, $\mathrm{SX}(\mathrm{t})$.

rhodopruinosum, Polymeridium: 633, 634(f), 929(f), 935(k), SX(t).

rhodotitthon, Cryptothelium (=Astrothelium purpurascens): 847(f), 882.

Riddlea (=Bathelium ): 895.

rimosum, Astrothelium: 626(f), 628, $800(\mathrm{k}), 827(\mathrm{f})$, $\mathrm{SX}(\mathrm{t})$.

rivana, Dacampiosphaeria: 977.

robusta, Aptrootia: 743(t), 752(p), 777(f), 779(f), 789(k), 790(f), 791, 791, 988, SA(a), SX(t).

robusta, Laurera (=Aptrootia robusta): 791 .

robustosporum, Astrothelium: 706, 707(f), 708(f), $811(\mathrm{k}), 840(\mathrm{f}), \mathrm{SX}(\mathrm{t})$.

robustum, Astrothelium: 729(t), 730(p), 745(t), 754(p), 811(k), 840(f), 883, SA(a), SC(p), SX(t).

rubescens, Trypethelium (= Pyrenula cruenta): 978.

rubrocinctum, Trypethelium (= Trypethelium plicatorimosum): 971 .

rubrocrystallinum, Astrothelium: 719(f), 719, 798(k), 824(f), SX(t).

rubroplatystomum, Trypethelium: $747(\mathrm{t}), 750(\mathrm{p})$, $\mathrm{SA}(\mathrm{a}), S B(\mathrm{p}), S C(\mathrm{p})$.

rubrostoma, Distothelia: 924(f), 927(k), 927, SX(t).

rubrostoma, Mycomicrothelia (= Distothelia rubrostoma): 927. rubrum, Trypethelium (= Pyrenula cruenta): 978.

rufescens, Astrothelium: 745(t), 752(p), 766(f), 771(f), 783(t), 801(k), 828(f), 883, SA(a), SC(p), SX(t).

salicicola var. minor, Massarina (= Nigrovothelium tropicum): 931.

salicinicola, Massaria (=Nigrovothelium tropicum): 931. salicinicola, Massarina (=Nigrovothelium tropicum): 931. sanguinaria, Laurera (=Astrothelium sanguinarium): 884 . sanguinarium, Astrothelium: 671, 745(t), 752(p), 780(f), 781, 813(k), 842(f), 884, SA(a), $S B(\mathrm{p}), S C(\mathrm{p})$, $\mathrm{SX}(\mathrm{t})$.

sanguinarium, Meristosporum (=Astrothelium sanguinarium): 884 .

sanguineoxanthum, Astrothelium: 626(f), 629, 781, 811(k), 841(f), SX(t).

santessonii, Astrothelium: $819(\mathrm{k}), 848(\mathrm{f}), \mathbf{8 8 4}, \mathrm{SX}(\mathrm{t})$. santessonii, Laurera (=Astrothelium santessonii): 884 .

saxicola, Astrothelium: $820(\mathrm{k}), 849(\mathrm{f}), 884,888$, $\mathrm{SX}(\mathrm{t})$.

saxicola, Cryptothelium (=Astrothelium saxicola): 885 .

saxicola, Pseudopyrenula: 885, 888, 954(k), 957(f), 959, $\mathrm{SX}(\mathrm{t})$.

schizostomoides, Thelotrema: 978.

schizostomum, Trypethelium (= Thelotrema schizostomoides): 978.

scitulens, Trypethelium (= Trypethelium eluteriae): 964.

sclerotium, Pertusaria: 978.

sclerotium, Trypethelium (= Pertusaria sclerotium): 978.

scoria f. endochraceum, Trypethelium (=Astrothelium luridum): 867.

scoria var. convexum, Trypethelium (=Astrothelium bicolor): 847.

scoria var. feei, Trypethelium (=Astrothelium feei): 860 .

scoria var. janeirense, Trypethelium (=Astrothelium bicolor): 848.

scoria var. phlyctaena, Trypethelium (= Astrothelium phlyctaena): 877.

scoria var. sordidius, Trypethelium (=Astrothelium scoria): $828(\mathrm{f}), 885$.

scoria, Astrothelium: 625, 674, 687(t), 745(t), 752(p), 783(t), 784, 800(k), 828(f), 850, 871, 885, SA(a), $S C(\mathrm{p}), \mathrm{SX}(\mathrm{t})$.

scoria, Pseudopyrenula (=Astrothelium scoria): 885.

scoria, Trypethelium (=Astrothelium scoria): 885 .

scorioides, Astrothelium: 687(t), 729(t), 730(p), 784, 808(k), 837(f), 886, 886, SX(t).

scorioides, Trypethelium (=Astrothelium scoriothelium): 886 .

scoriothelium, Astrothelium: $801(\mathrm{k}), 830(\mathrm{f}), \mathbf{8 8 6}$, $\mathrm{SX}(\mathrm{t})$.

scorites, Trypethelium (= Viridothelium virens): 975.

scorizum, Astrothelium: 745(t), 752(p), 773(f), 783(t), 808(k), 838(f), 886, SA(a), SX(t).

scorizum, Trypethelium (=Astrothelium scorizum): 886 .

seminudum, Architrypethelium (=Architrypethelium nitens): 794.

seminudum, Pyrenastrum (=Architrypethelium nitens): 794 .

septemseptatum, Astrothelium: 629, 630(f), 806(k), 835(f), SX(t).

septicollare, Astrothelium (= Pyrenula septicollaris): 977.

septicollaris, Pyrenula: 977.

sepulta, Heufleria (=Astrothelium sepultum): 886 .

sepulta, Laurera (=Astrothelium sepultum): 886 .

sepultum, Astrothelium: 644, 651, 687(t), 745(t), 752(p), 817(k), 846(f), 886, SA(a), SC(p), SX(t).

sepultum, Cryptothelium (=Astrothelium sepultum): 795, 886.

seriale, Sclerophyton: 978 .

sericea, Polyblastiopsis: 751 . 
serusiauxii, Pseudopyrenula: 955(k)，956(f)，959, $\mathrm{SX}(\mathrm{t})$.

sexloculatum, Astrothelium: 630(f), 631, 806(k), 835(f), $\mathrm{SX}(\mathrm{t})$.

siamense, Astrothelium: 728, 729(t), 730(p), 734, 735(f), 735, 806(k), 835(f), SX(t).

siamense, Polymeridium: 935(k), 950, SX(t).

siamensis, Arthopyrenia (= Polymeridium siamense): 950.

sierraleonense, Astrothelium: 780(f), 812(k), 842(f), $887, \mathrm{SX}(\mathrm{t})$.

sierraleonense, Tremotylium (=Astrothelium sierraleonense): 887 .

sikkimense, Astrothelium: 814(k), 843(f), 887, SX(t). sikkimensis, Laurera (=Astrothelium sikkimense): 887.

simplex, Astrothelium: 716, 719(f), 720, 808(k), 837(f), $\mathrm{SX}(\mathrm{t})$.

simplicatum, Astrothelium (= Astrothelium laevigatum): 833(f), 866.

simulans, Polymeridium: 773(f), 935(k), 939(f), 950, $\mathrm{SX}(\mathrm{t})$.

sinuosum, Astrothelium: 719(f), 720, 720, 722(f), 806(k), 835(f), SX(t).

sipmanii, Astrothelium: 630(f), 631, 696, 719, 803(k), 831(f), SX(t).

sitiana, Pseudopyrenula (= Pseudopyrenula diluta): 957(f), 957.

socialis, Bogoriella: 902, 903(k), 904(k), 906(f), 915, $\mathrm{SX}(\mathrm{t})$.

socialis, Microthelia (=Bogoriella socialis): 915.

socialis, Mycomicrothelia (=Bogoriella socialis): 915 .

solitarium, Astrothelium: 707(f), 708(f), 709, $801(\mathrm{k})$, 830(f), SX(t).

solomonense, Viridothelium: 634(f), 637, 811(k), 973(k), 974(f), SX(t)

sordidescens, Trypethelium (=Leucodecton compunctum): 978.

sordithecium, Astrothelium: 652(f), 653, 805(k), 833(f), $\mathrm{SX}(\mathrm{t})$.

speciosum, Astrothelium (=Anthracothecium speciosum): 977.

spectabile, Astrothelium: 803(k), 831(f), 887.

spectabile, Trypethelium (=Astrothelium spectabile): 887 .

sphaerica, Laurera (=Bathelium sphaericum): 901.

sphaerica, Polyblastiopsis (=Bathelium sphaericum): 901.

sphaericum, Bathelium: 678, 813(k), 896(k), 898(f), 901, $\mathrm{SX}(\mathrm{t})$.

sphaerioides, Astrothelium: 772(f), 816(k), 845(f), $888, \mathrm{SX}(\mathrm{t})$

sphaerioides, Bathelium (=Astrothelium sphaerioides): 888 . sphaerioides, Laurera (=Astrothelium sphaerioides): 888 .

sphaerioides, Trypethelium (=Astrothelium sphaerioides): 888 .

sphaerocephala, Pseudopyrenula (= Trypethelium sphaerocephalum): 971.

sphaerocephalum, Trypethelium: 753, 964(k), 968(f), 971, SX(t).

sprengelii $\mathrm{f}$. inaequale, Trypethelium (= Trypethelium inaequale): 969.

sprengelii var. anacardii, Trypethelium (=Trypethelium eluteriae): 964.

sprengelii var. citrinum, Trypethelium (= Trypethelium eluteriae): 964.

sprengelii var. nigricans, Trypethelium (= Trypethelium eluteriae): 964

sprengelii var. porosa, Trypethelium (=Astrothelium porosum): 879. sprengelii, Trypethelium (= Trypethelium eluteriae): $748(\mathrm{t})$, $750(\mathrm{p}), 964,966(\mathrm{f}), 967, \mathrm{SA}(\mathrm{a}), S C(\mathrm{p})$.

sprucei, Tremotylium (=Marcelaria purpurina): 929(f), 930.

staphyleae, Massarina (= Pseudopyrenula staphyleae): 959.

staphyleae, Pseudopyrenula: 955(k), 959, SX(t).

stirtoniana, Melanotheca (=Pyrenula): 978.

stirtonianum, Trypethelium (= Pyrenula sp.): 978.

straminea, Clathroporina (=Astrothelium stramineum): 888.

straminea, Laurera (=Astrothelium stramineum): 888.

straminea, Thelenella (=Astrothelium stramineum): 888 .

stramineoatra, Arthopyrenia (=Polymeridium stramineoatrum): 950.

stramineoatrum, Polymeridium: 934(k), 950, SX(t).

stramineum, Astrothelium: 811(k), 841(f), 885, 888, $888, \mathrm{SX}(\mathrm{t})$.

stramineum, Trypethelium (=Astrothelium straminicolor): 837(f), 888.

straminicolor, Astrothelium: 774(f), 783(t), 808(k), 837(f), 888, SX(t).

straminicolor, Trypethelium (=Astrothelium straminicolor): 888.

striguloides, Bogoriella: 903(k), 906(f), 915, 988, SX(t). striguloides, Mycomicrothelia (=Bogoriella striguloides): 915.

stromatofluorescens, Astrothelium: 687(t), 707(f), 708(f), 709, 783(t), 797(k), 823(f), SX(t).

subaequans, Astrothelium: $818(\mathrm{k}), 847(\mathrm{f}), 889, \mathrm{SX}(\mathrm{t})$.

subalbens, Bathelium (=Astrothelium phlyctaena): 878 .

subalbens, Trypethelium (=Astrothelium phlyctaena): 823(f), 878.

subbenguelensis, Laurera (=Marcelaria benguelensis): 928.

subcatervarium, Astrothelium: 745(t), 752(p), 799(k), 825(f), 889, SA(a), SX(t).

subcatervarium, Trypethelium (=Astrothelium subcatervarium): 889.

subcinerea, Porina (= Polymeridium subcinereum): 951.

subcinerea, Pyrenula (= Polymeridium subcinereum): 951.

subcinerea, Verrucaria (= Polymeridium subcinereum): 951.

subcinereum, Polymeridium: 688(t), 769(f), 773(f), 785, 936(k), 939(f), 951, SX(t).

subclandestinum, Astrothelium: 709, 710, $810(\mathrm{k})$, 839(f), 889, SX(t).

subdiscreta, Laurera (=Astrothelium subdiscretum): 890 .

subdiscretum, Astrothelium: 813(k), 843(f), 890, SX(t). subdiscretum, Bathelium (=Astrothelium subdiscretum): 890 .

subdiscretum, Trypethelium (=Astrothelium subdiscretum): 890.

subdisjuncta, Bottaria (=Astrothelium subdisjunctum): 890 . subdisjuncta, Laurera (=Astrothelium subdisjunctum): 890 . subdisjunctum, Astrothelium: 665, 816(k), 845(f), $890, \mathrm{SX}(\mathrm{t})$.

subdissocians, Astrothelium: $809(\mathrm{k}), 838(\mathrm{f}), 891, \mathrm{SX}(\mathrm{t})$. subdissoicans, Astrothelium: 783(t).

subeluteriae, Trypethelium: $636,685,686,748(\mathrm{t})$, 750(p), 753, 755, 773(f), 779(f), 785, 963(k), 967(f), 972, $\mathrm{SA}(\mathrm{a}), S B(\mathrm{p}), S C(\mathrm{p}), S \mathrm{~S}(\mathrm{t})$.

subendochryseum, Astrothelium: 652(f), 653, 745(t), 752(p), 801(k), 829(f), SA(a), SC(p), SX (t).

subfallens, Bogoriella: $688(\mathrm{t}), 785,904(\mathrm{k}), 906(\mathrm{f})$, 915, $\mathrm{SX}(\mathrm{t})$

subfallens, Microthelia (=Bogoriella subfallens): 915.

subfallens, Mycomicrothelia (= Bogoriella subfallens): 915.

subfallens, Verrucaria (=Bogoriella subfallens): 915. 
subfuscum, Astrothelium: 783(t), 809(k), 838(f), 891, $\mathrm{SX}(\mathrm{t})$.

subgregaria, Pseudopyrenula: 635, 689(t), 711, 747(t), $750(\mathrm{p}), 785,954(\mathrm{k}), 956(\mathrm{f}), 959,960,961, \mathrm{SA}(\mathrm{a})$, $S B(\mathrm{p}), S C(\mathrm{p}), \mathrm{SX}(\mathrm{t})$.

subimitans, Arthopyrenia (=Polymeridium subcinereum): 951. subincruentum, Trypethelium (= Pyrenula cruenta): 978.

subinterjectum, Astrothelium: 652(f), 654, 745(t), 752(p), 783(t), 805(k), 834(f), SA(a), $S B(\mathrm{p}), S C(\mathrm{p})$, $\mathrm{SX}(\mathrm{t})$.

submadreporiformis, Laurera (=Bathelium mastoideum): 900. submuriforme, Polymeridium: 937(k), 941(f), 951, $\mathrm{SX}(\mathrm{t})$.

subnitidiusculum, Trypethelium (= Trypethelium epileucodes): 969.

subnudata, Pseudopyrenula: 636, 689(t), 747(t), $750(\mathrm{p}), 772(\mathrm{f}), 785,953,954(\mathrm{k}), 957(\mathrm{f}), 960,960$, 989, SA(a), SX(t).

suboculata, Arthopyrenia (=Polymeridium sulphurescens): 952. subpersicina, Bogoriella (=Bogoriella decipiens): 902, 907(f), 909.

subphaeomelodes, Laurera (=Astrothelium subdiscretum): 890. subplanum, Trypethelium (=Anthracothecium gregale): 978 .

subscoria, Astrothelium: 669(f), 670(f), 674, 688(t), 745(t), 746(t), 752(p), 766(f), 783(t), 800(k), 828(f), $\mathrm{SA}(\mathrm{a}), S B(\mathrm{p}), S C(\mathrm{p}), S \mathrm{X}(\mathrm{t})$.

subsphaerioides, Laurera (=Astrothelium cf. subdiscretum): 843(f), 976.

subsulphureum, Trypethelium (= Trypethelium eluteriae): 964 . subumbilicata, Pyrenula: 978.

subumbilicatum, Trypethelium (= Pyrenula subumbilicata): 978.

subvariata, Heufleria (=Astrothelium variatum): 845(f), 893. subvariatum, Cryptothelium (=Astrothelium variatum): 893 . subvariatum, Trypethelium (=Astrothelium variatum): 893 . subvariolosa, Pyrenula: 978.

subvariolosum, Astrothelium (=Astrothelium stramineum): 837(f), 888.

subvelata, Arthopyrenia: 978.

subvelata, Pseudopyrenula (=Arthopyrenia subvelata): 978.

subvirescens, Polymeridium: 688(t), 747(t), 750(p), 932(f), 934(k), 951, SA(a), SC(p), SX(t).

subvirescens, Porina (= Polymeridium subvirescens): 951.

subvirescens, Verrucaria (=Polymeridium subvirescens): 951.

suffusa, Arthopyrenia (= Polymeridium suffusum): 952.

suffusa, Verrucaria (=Polymeridium suffusum): 952.

suffusum, Polymeridium: 929(f), 935(k), 952, SX(t). sulphurescens, Arthopyrenia (=Polymeridium sulphurescens): 952.

sulphurescens, Polymeridium: 936(k), 939(f), 952, 952, SX(t).

sulphurescens, Pseudopyrenula (= Polymeridium sulphurescens): 952.

sulphureum (Eschw.) Müll. Arg., Astrothelium (=Astrothelium pyrenastrosulphureum): 883.

sulphureum Nyl., Astrothelium (=Astrothelium pyrenastrosulphureum): 883.

sulphureum subsp. plicatum, Pyrenastrum (=Astrothelium pyrenastrosulphureum): 883.

sulphureum var. macrocarpon, Astrothelium (=Astrothelium macrocarpum): 867. sulphureum var. subpallescens, Astrothelium (=Astrothelium pyrenastrosulphureum): 883.

sulphureum, Pyrenastrum (=Astrothelium pyrenastrosulphureum): 883.

sundaica, Laurera (= Trypethelium epileucodes): 969.

sundaicum, Bathelium (= Trypethelium epileucodes): 969.

superans, Parathelium (=Architrypethelium nitens): 794.

superans, Pseudopyrenula: 685, 954(k), 955(k), 956(f), 961, SX (t).

superans, Splanchnonema (=Architrypethelium nitens): 794 .

superbum, Astrothelium: $820(\mathrm{k}), 891, \mathrm{SX}(\mathrm{t})$.

superbum, Campylothelium (=Astrothelium superbum): 891 . superbum, Trypethelium (=Astrothelium superbum): 891 .

supraclandestinum, Astrothelium: 696, 707(f), 708(f), 709, $810(\mathrm{k}), 840(\mathrm{f}), \mathrm{SX}(\mathrm{t})$.

tenue, Astrothelium: $819(\mathrm{k}), 848(\mathrm{f}), 891, \mathrm{SX}(\mathrm{t})$.

tenue, Campylothelium (=Astrothelium tenue): 892 .

terricola, Aptrootia: 743(t), 752(p), 777(f), 779(f), 789(k), 789, 790(f), 791, 791, SA(a), SX(t).

terricola, Thelenella (=Aptrootia terricola): 791 .

tessella, Pseudopyrenula (=Astrothelium phlyctaena): 877.

tessella, Verrucaria (=Astrothelium phlyctaena): 877.

testudineum, Astrothelium: 707(f), 710, 820(k), 849(f), $\mathrm{SX}(\mathrm{t})$.

tetracerae var. crocea, Verrucaria (=Astrothelium aeneum): 821 . tetraspora, Didymosphaeria (=Bogoriella obovata): 913 .

tetrasporum, Astrothelium: 719(f), 721, 815(k), 845(f), $\mathrm{SX}(\mathrm{t})$.

tetrathalamia, Pertusaria: 978.

tetrathalamium, Trypethelium (= Pertusaria tetrathalamia): 978.

thallina, Pseudopyrenula: 635, 954(k), 956(f), SX(t). thaxteri var. heterogena, Thelenella (=Phyllobathelium chlorogastricum): 976.

thaxteri, Phyllobathelium (=Phyllobathelium chlorogastricum): 976.

thaxteri, Thelenella (= Phyllobathelium chlorogastricum): 976.

thelena var. subtriseptata, Microthelia (=Bogoriella thelena): 916 .

thelena, Bogoriella: 904(k), 905(k), 907(f), 908, 916, $\mathrm{SX}(\mathrm{t})$.

thelena, Microthelia (=Bogoriella thelena): 916.

thelena, Mycomicrothelia (=Bogoriella thelena): 916.

thelena, Verrucaria (=Bogoriella thelena): 916.

thelenoides, Didymosphaeria (=Bogoriella obovata): 913.

thelotremoides, Astrothelium: 797(k), 824(f), 892, $\mathrm{SX}(\mathrm{t})$.

thelotremoides, Pseudopyrenula (=Astrothelium thelotremoides): 892.

thelotremoides, Trypethelium (=Astrothelium thelotremoides): 892.

thelotremoides, Verrucaria (=Astrothelium thelotremoides): 892.

tolimense, Trypethelium: 656(f), 657, 962(k), SX(t).

triangularis, Bogoriella: 903(k), 906(f), 916, SX(t).

triangularis, Mycomicrothelia (=Bogoriella triangularis): 916.

tribulationis, Polymeridium: 929(f), 935(k), 952, SX(t). tricolor, Trypethelium (=Astrothelium pulcherrimum): 823(f), 881.

tricolor, Viridothelium: 656(f), 657, 748(t), 752(p), $820(\mathrm{k}), 973(\mathrm{k}), 974(\mathrm{f}), \mathrm{SA}(\mathrm{a}), S C(\mathrm{p}), \mathrm{SX}(\mathrm{t})$.

triplex, Trypethelium (=Astrothelium phlyctaena): 878 . 
tristis, Verrucaria (= Nigrovothelium tropicum): 931. tropica, Pseudopyrenula (= Nigrovothelium tropicum): 757, 931. tropica, Pyrenula (= Nigrovothelium tropicum): 757, 931. tropica, Sagedia (= Nigrovothelium tropicum): 757, 931. tropica, Verrucaria (=Nigrovothelium tropicum): 757, 931. tropicum var. nigratum, Trypethelium (= Nigrovothelium tropicum): 931.

tropicum, Nigrovothelium: 624, 655, 688(t), 747(t), $750(\mathrm{p}), 757,757,769(\mathrm{f}), 772(\mathrm{f}), 775,776(\mathrm{f}), 784$, 801(k), 930, 931(k), 931, 932(f), 989, SA(a), $S B(\mathrm{p})$, $S C(\mathrm{p}), \operatorname{SX}(\mathrm{t})$.

tropicum, Spermatodium (= Nigrovothelium tropicum): 757 , 931.

tropicum, Trypethelium (= Nigrovothelium tropicum): 656, 748, 749, 753, 757, 931, 962.

trypetheliiformis, Verrucaria (=Trypethelium eluteriae): 964.

trypethelioides, Astrothelium: 630(f), 631, $810(\mathrm{k})$, 839(f), SX(t).

Trypethelium: $640,643,658,741,748,749(\mathrm{p}), 749$, 750 (p), 753, 755, 758, 759, 765, 775, 778, 782, 786, 787, 788(k), 792, 794, 796(k), 895, 961, 972.

trypethelizans, Astrothelium: 803(k), 831(f), 892, SX(t). trypethelizans, Pseudopyrenula (=Astrothelium trypethelizans): 892.

trypethelizans, Trypethelium (=Astrothelium trypethelizans): 892.

trypethelizans, Verrucaria (=Astrothelium trypethelizans): 892.

tuberculosa, Laurera (= Bathelium tuberculosum): 901.

tuberculosum, Astrothelium: 668, 746(t), 754(p), 769(f), 771(f), 775, 776(f), 802(k), 830(f), 893, $\mathrm{SA}(\mathrm{a}), \mathrm{SX}(\mathrm{t})$.

tuberculosum, Bathelium: 746(t), 752(p), 812(k), 896(k), 898(f), 901, SA(a), SC(p), SX(t).

tuberculosum, Trypethelium (=Astrothelium tuberculosum): 893.

tumida, Arthopyrenia (= Polymeridium catapastum): 944. uberina, Pertusaria (=Architrypethelium uberinum): 794. uberina, Porina (=Architrypethelium uberinum): 794 .

uberina, Porophora (=Architrypethelium uberinum): 794 .

uberina, Pseudopyrenula (=Architrypethelium uberinum): 794. uberina, Pyrenula (=Architrypethelium uberinum): 794 . uberina, Verrucaria (=Architrypethelium uberinum): 794 . uberinoides, Trypethelium (=Architrypethelium uberinum): 794 . uberinum, Architrypethelium: 743(t), 752(p), 790(f), 792(k), 792, 793, 794, 977, SA(a), SX(t).

uberinum, Stromatothelium (=Architrypethelium uberinum): 794.

uberinum, Trypethelium (=Architrypethelium uberinum): 794 . ubianense, Astrothelium: $802(\mathrm{k}), 831(\mathrm{f}), 893, \mathrm{SX}(\mathrm{t})$. ubianense, Trypethelium (=Astrothelium ubianense): 893. ubianensis, Pseudopyrenula (=Astrothelium ubianense): 893. ultralucens, Astrothelium: 630(f), 632, 805(k), 834(f), $\mathrm{SX}(\mathrm{t})$.

umbilicatum, Astrothelium: 977.

umbilicatum, Trypethelium (= Pyrenula subvariolosa): 978 . usambarensis, Holstiella (= Trypethelium eluteriae): 961, 964. varia, Laurera (= Astrothelium deforme): 856.

varia, Meissneria (=Astrothelium deforme): 795, 856.

variabile, Astrothelium: 674, 675(f), 676(f), 677, 688(t), 812(k), 841(f), SX(t).

variata, Laurera (=Astrothelium variatum): 893. variatum, Astrothelium: $815(\mathrm{k}), 845(\mathrm{f}), \mathbf{8 9 3}, \mathrm{SX}(\mathrm{t})$. variatum, Trypethelium (=Astrothelium variatum): 893 . variolosum, Astrothelium: 653, 655, 688(t), 728, 734, 735, 736, 746(t), 754(p), 766, 782, 783(t), 785, 805(k), 834(f), 893, SA(a), SB(p), SC(p), SX(t). variolosum, Trypethelium (=Astrothelium variolosum): 893 . varium var. citrinum, Astrothelium (= Trypethelium eluteriae): 964.

varium, Astrothelium (= Trypethelium eluteriae): 964 . varium, Bathelium (=Astrothelium deforme): 856. varium, Trypethelium (=Astrothelium deforme): 856 . velata, Laurera (=non-lichenized fungus): 977. velatum, Bathelium (=non-lichenized fungus): 977. verrucarioides, Arthopyrenia (= Polymeridium pyrenuloides): 949.

verrucarioides, Chiodecton (= Enterographa verrucarioides): 978.

verrucarioides, Enterographa: 978.

verrucarioides, Trypethelium (=Enterographa verrucarioides): 978.

verrucoaggregata, Laurera (=Astrothelium subdiscretum): 843(f), 890.

verrucosa, Pseudopyrenula (=Nigrovothelium tropicum): 931.

verrucosum (Vain.) Zahlbr., Trypethelium (=Nigrovothelium tropicum): 931.

verrucosum Fée, Trypethelium (= Pertusaria sp.): 979.

versicolor, Astrothelium: 673, 688(t), 771(f), 784, 804(k), 833(f), 843, 862, 894, SX(t).

vezdae, Laurera (=Astrothelium vezdanum): 894 .

vezdana, Laurera (=Astrothelium vezdanum): 894 .

vezdanum, Astrothelium: 813(k), 842(f), 894, SX(t).

violascens, Trypethelium (=Astrothelium pleiostomum): 839(f), 879.

virens, Trypethelium (=Viridothelium virens): 748, 749, 758, 759, 975.

virens, Viridothelium: 658, 659, 748(t), 752(p), 758, 759, 784, 785, 802(k), 962, 963(k), 969, 972, 973(k), 974(f), 975, SA(a), SX(t).

virginicum, Trypethelium (= Trypethelium epileucodes): 967(f), 969.

Viridothelium: 748, 749(p), 749, 753, 758, 758, 778, 787, 788(k), 789(k), 879, 962, 972.

vonkonratii, Viridothelium: 656(f), 658, 748(t), 752(p), 811(k), 973(k), 974(f), SA(a), SX(t).

vulcanum, Astrothelium: 630(f), 632, 783(t), 797(k), 822(f), SX(t).

wainiana, Clathroporina (= Astrothelium subdisjunctum): 890 . willeyana, Mycomicrothelia (=Bogoriella socialis): 902, 915.

xanthoexcentricum, Polymeridium: 682(f), 683, 684(f), $685,688(\mathrm{t}), 936(\mathrm{k}), 940(\mathrm{f}), \mathrm{SX}(\mathrm{t})$.

xanthonica, Bogoriella: 903(k), 912, 916, SX(t).

xanthonica, Mycomicrothelia (=Bogoriella xanthonica): 916.

xanthoplatystomum, Trypethelium: 657, 682(f), 684(f), 686, 689(t), 689, 962(k), 965(f), SX(t).

xanthoreagens, Polymeridium: 929(f), 934(k), 952, SX(t).

xanthosuperbum, Astrothelium: 679, 707(f), 711, 812(k), 841(f), SX(t).

zahlbruckneri, Campylothelium (=Astrothelium megaspermum): 870.

zebrinum, Astrothelium: 633, 634(f), $810(\mathrm{k}), 840(\mathrm{f})$, $\mathrm{SX}(\mathrm{t})$.

zenkeriana, fulella (=Astrothelium megaspermum): 870 . 